hep-th/0306170

SU-ITP-03-16

\title{
The Black Hole Singularity in AdS/CFT
}

\author{
Lukasz Fidkowski, Veronika Hubeny, Matthew Kleban, and Stephen Shenker \\ Department of Physics, Stanford University, Stanford, CA, 94305, USA
}

We explore physics behind the horizon in eternal AdS Schwarzschild black holes. In dimension $d>3$, where the curvature grows large near the singularity, we find distinct but subtle signals of this singularity in the boundary CFT correlators. Building on previous work, we study correlation functions of operators on the two disjoint asymptotic boundaries of the spacetime by investigating the spacelike geodesics that join the boundaries. These dominate the correlators for large mass bulk fields. We show that the Penrose diagram for $d>3$ is not square. As a result, the real geodesic connecting the two boundary points becomes almost null and bounces off the singularity at a finite boundary time $t_{c} \neq 0$.

If this geodesic were to dominate the correlator there would be a "light cone" singularity at $t_{c}$. However, general properties of the boundary theory rule this out. In fact, we argue that the correlator is actually dominated by a complexified geodesic, whose properties yield the large mass quasinormal mode frequencies previously found for this black hole. We find a branch cut in the correlator at small time (in the limit of large mass), arising from coincidence of three geodesics. The $t_{c}$ singularity, a signal of the black hole singularity, occurs on a secondary sheet of the analytically continued correlator. Its properties are computationally accessible.

The $t_{c}$ singularity persists to all orders in the $1 / m$ expansion, for finite $\alpha^{\prime}$, and to all orders in $g_{s}$. Certain leading nonperturbative effects can also be studied. The behavior of these boundary theory quantities near $t_{c}$ gives, in principle, significant information about stringy and quantum behavior in the vicinity of the black hole singularity.

June, 2003 


\section{Introduction}

Some of the deepest mysteries in quantum gravity lie hidden behind horizons, including the nature of the spacelike singularities inside black holes. A better understanding of these may shed more light on cosmological singularities as well. Despite much recent

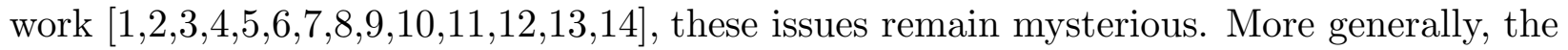
existence of horizons and resolving the puzzles of information loss and quantum decoherence require that the description of physics inside the horizon be linked somehow to the description outside. This connection-the central concept of black hole complementarity [15, [16]-is still not well understood.

As observations continue to bolster the case for cosmological inflation, this set of questions becomes more pressing. A complete description of eternal inflation [17] probably requires an understanding of the many "universes" behind the de Sitter horizon of a single inflating patch [18,19,20].

Despite the enormous progress in our understanding of quantum gravity in recent years, physics behind the horizon remains extremely puzzling. This is due in part to the holographic [15,21] nature of our most fully developed nonperturbative descriptions of quantum gravity, the brane bulk correspondences of Matrix Theory [22] and AdS/CFT [23], [24,25,26]. These formulations describe physics from the point of view of an observer outside the horizon. For example, CFT correlators in AdS/CFT describe observables on the asymptotic spatial boundary of AdS space. In the thermal CFT that describes a large black hole in AdS/CFT [27], these asymptotic observables apparently describe physics only outside the horizon.

The brane bulk correspondences provide a fully self consistent quantum description of gravitational dynamics outside of the horizon, demonstrating that quantum coherence is not lost. But these descriptions appear to have little to say about behind the horizon phenomena.

However, this is not the whole story. A number of authors have explored ways to extract behind the horizon information from boundary correlators [28, 29, 30, 31, 32]. 10 In particular Maldacena [30] argued that the boundary description of the eternal Anti-de Sitter Schwarzschild black hole consisting of one copy of the CFT on each of the two asymptotic boundaries could give some information about physics behind the horizon. In this approach, the one boundary thermal description is recovered by tracing over the Hilbert space of the other boundary CFT as in the real-time or thermofield formalism for thermal field theory [44]. The thermal state counting entropy arises from the entanglement

1 There is also large body of work addressing more general questions of extracting behind the horizon physics and holographic representation of horizons, as well as of generic local objects in the bulk; see e.g. [33, 34, 35, 36, 37, 38, 39, 40, 41, 42, 43, 
entropy of the pure entangled "Hartle-Hawking" state in which expectation values are computed. This realizes an old idea of Israel [45].

These ideas were explored in some detail for $d=3$, the BTZ black hole, in 31. The finiteness of amplitudes (manifest from the boundary or bulk Euclidean point of view) was shown to arise in the bulk from $i \epsilon$ regulation of the metric singularity inherent in analytic continuation from the Euclidean theory and from cancellations between future and past singularities.

In addition, [31] gave a simple demonstration of how boundary correlation functions can probe physics behind the horizon by studying the correlator of two operators, one on each asymptotic boundary, each creating a large mass bulk particle. As the mass $m \rightarrow \infty$, the correlator can be evaluated in the semiclassical geodesic approximation and is given by $\exp (-m \mathcal{L})$. Here $\mathcal{L}$ is the proper length of the spacelike geodesic joining the boundary points (see Fig.5a). Because the geodesic passes through spacetime regions inside the horizon, this boundary correlator reveals information about the geometry behind the horizon. This closely parallels a prior calculation done in the "geon" geometry [29].

As for all such quantities, an "outside the horizon" interpretation can be given. By tracing the Hartle-Hawking state, perturbed by the operator on one boundary, over the Hilbert space of the CFT on that boundary, a perturbed density matrix for the remaining CFT is created. This density matrix corresponds in the bulk to a modified boundary condition on the horizon, which can have nontrivial correlations with an asymptotic boundary field. The nature of this boundary condition is obscure from the "outside the horizon" point of view, but natural when physics behind the horizon is taken into account.

The BTZ geodesics illustrated in Fig.5a pass quite close to the singularity for moderate boundary times. But the correlation function is relatively structureless. Presumably this reflects the simple orbifold geometry of the BTZ black hole. The curvature of the BTZ geometry is constant except for a delta function at the singularity.

Our goal in this paper is to explore further what information from behind the horizon can be found in boundary AdS/CFT correlators. To consider a situation with a more interesting geometry, we focus on AdS Schwarzschild black holes in $d>3$, and in particular in $d=5$ where the boundary CFT is four dimensional $\mathcal{N}=4$ super Yang Mills theory. The geometry near the singularity in such black holes approaches that of $d>3$ Schwarzschild, and so the curvature diverges as one approaches the singularity. This affects the geodesics dramatically.

We begin in Section 2 by exploring in detail the causal structure and spacelike geodesics in $d=5 \mathrm{AdS}$ Schwarzschild. (Other dimensions greater than three behave similarly.) We find several unusual features. First, the Penrose diagram that encodes the causal structure of the geometry is not a square, unlike the $d=3$ case. The lines describing the singularity bend inward toward the center of the diagram. This allows a nearly 
null geodesic beginning at the boundary at a finite nonzero time $t_{c}$ to "bounce" off the singularity and hit the other boundary at the symmetric point. This behavior, which does not occur for $d=3$, is depicted in Fig.5b.

After submitting this paper we have learned that such features have previously been found in a general study of effectively two dimensional metrics 46] 2 .

In Section 3 we turn to correlators in the geodesic approximation. If the bounce geodesic were to dominate, the correlator would become singular as $t \rightarrow t_{c}$ because the proper distance goes to zero as the geodesic becomes null. This would be a kind of "light cone singularity." But general considerations about the boundary field theory rule out this kind of behavior. In fact this geodesic does not dominate the correlator. There are in general multiple geodesics that connect the two boundary points (Fig.12). At $t=0$ their proper distances coincide, creating a branch point in the correlator which behaves as $t^{4 / 3}$ for small $t$. By studying various resolutions of this branch point, we show that as $t$ increases from 0 , the correlator defined by the boundary CFT is given by a symmetric sum of the two complex branches of this expression. Each of these can be attributed to a complex geodesic in complexified spacetime. At large $t$ these complex branches reproduce the correct quasinormal modes for this black hole, which are complex, unlike the $d=3$ case.

But the correlator is an analytic function of $t$ and can be continued onto the real sheet. (This can be done in a computationally effective manner, as discussed in Appendix F.) On this sheet, the "light cone singularity" does appear. So the boundary correlator does contain information about the singularity, albeit in a subtle way.

In Section 4 we extend the above analysis to include finite $m$, finite string length $l_{s}\left(\alpha^{\prime} \sim l_{s}^{2}\right)$, and finite string coupling $\left(g_{s}\right)$ effects. We take wrapped D-branes (giant gravitons [47]) as explicit examples of heavy probe particles. The branch point at $t=0$ is smoothed out for any finite $m$, preventing analytic continuation to the bouncing geodesic. But at each order in the $1 / m$ expansion the branch point persists and one can follow this geodesic and the accompanying fluctuation corrections into the bouncing domain. The $1 / m$ corrections are given by a heat kernel expansion with coefficients related to the curvature and are large when the geodesic passes near the singularity. These quantities give a clear example of information about the singular geometry being coded into boundary CFT correlators.

The main corrections at finite but small $l_{s}$ (with $g_{s}$ kept equal to zero) can be expressed as modifications of the supergravity field equations and hence of the background metric, dilaton, etc. fields. Such small modifications do not change the basic picture outlined above. The branch point at $t=0$ either persists or, more generically, splits into two nearby branch points. In either case we can follow the geodesic onto the real sheet. Of

2 We are grateful to Jorma Louko for bringing this reference to our attention. 
course, for $t$ near $t_{c}$, we expect the $l_{s}$ corrections to become large because the geodesic passes through regions of large curvature.

At large $l_{s}$ the 't Hooft coupling $\lambda$ in the boundary gauge theory becomes small. In weak coupling perturbative gauge theory amplitudes are smooth at $t=0$. There is no branch point. Therefore, for consistency, there must be a phase transition at finite $\lambda$ (for infinite $m$ ).

Finite $g_{s}$ is a particularly difficult regime in which to study these phenomena. Wrapped D-brane masses are $\sim 1 / g_{s}$ and so are finite here. This smooths out the branch point and would seem to prevent analytic continuation to the $t_{c}$ singularity. But by taking appropriate $g_{s} \rightarrow 0$ limits we can extract behavior around the singularity to all orders in $g_{s}$, as well as study certain leading nonperturbative effects.

These results demonstrate that a significant amount of information from behind the horizon, and in particular from near the singularity, is encoded in boundary theory correlators. In Section 5 we discuss the meaning of these results further.

\section{Classical geometry}

We will devote this section to the exploration of the classical Schwarzschild-AdS geometry. As is well known, the AdS Schwarzschild spacetimes contain spacelike curvature singularities, event horizons, and timelike boundaries, all of which will play an important role in the discussion. First, we analyze the causal structure in detail, arriving at the surprising observation that, unlike the three dimensional case, for $d>3$ the Penrose diagram of AdS Schwarzschild spacetimes cannot be drawn as a square, with both boundaries and singularities represented by straight lines. Next, we study spacelike geodesics in this geometry, which, as we will discuss in the next section, provide a method for approximating the relevant boundary correlators.

AdS Schwarzschild represents a $d \geq 3$ dimensional, two-parameter family of solutions characterized by the size of the black hole and the AdS radius. For simplicity we will focus on the large black hole (planar) limit, where the AdS radius is much smaller than the horizon radius. Also, we will from now on concentrate on $d=5$. The geometry for finite-sized black holes, as well as in other dimensions $d>3$, is qualitatively similar. We will contrast this with the quite different case of the $d=3$ BTZ black hole [31.

The metric of the 5-dimensional AdS Schwarzschild spacetime is given by

$$
\begin{aligned}
d s^{2} & =-f(r) d t^{2}+\frac{d r^{2}}{f(r)}+r^{2} d \Omega_{3}^{2} \\
f(r) & =\frac{r^{2}}{R^{2}}+1-\frac{r_{+}^{2}}{r^{2}}\left(\frac{r_{+}^{2}}{R^{2}}+1\right),
\end{aligned}
$$




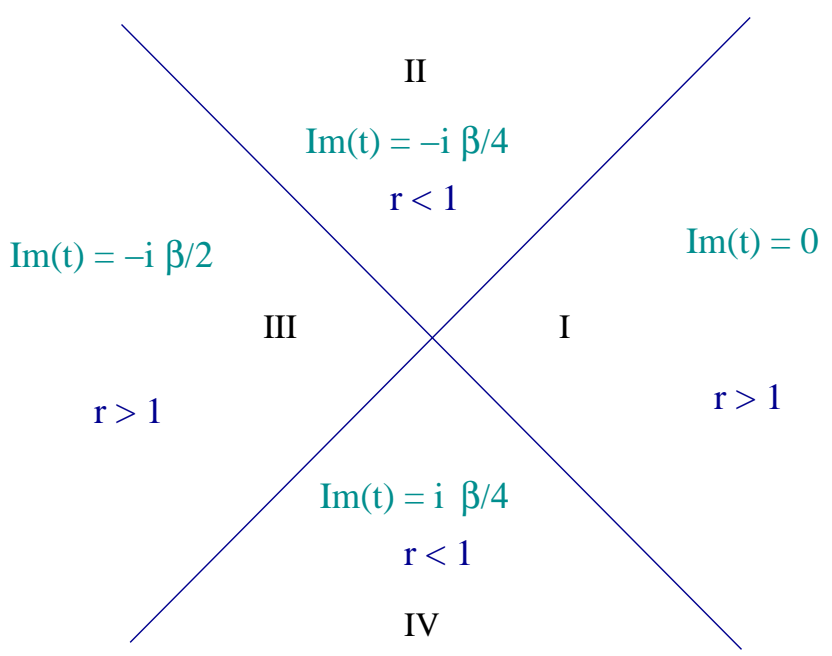

Fig. 1: Complexified coordinates for the AdS black hole: the time coordinate is complex in the extended spacetime, but with constant imaginary part in each wedge, as indicated above. The wedges are separated by the horizons at $r=1$, where $t$ diverges. Note that the real part of $t$ increases upward in wedge I, downward in wedge III, and to the right (left) in wedge II (IV).

where $r_{+}$is the horizon radius and $R$ is the AdS radius. We work in the limit of the infinitely massive black hole $r_{+} / R \rightarrow \infty$, where the metric simplifies. In particular, if we rescale the coordinates $r \rightarrow \frac{r_{+}}{R} r, t \rightarrow \frac{R}{r_{+}} t$, measure lengths in AdS units so $R=1$, and suppress the angular coordinates $\Omega$ that will not concern us here, the metric in the $t$ - $r$ plane becomes

$$
\begin{aligned}
& d s^{2}=-f(r) d t^{2}+\frac{d r^{2}}{f(r)} \\
& f(r)=r^{2}-\frac{1}{r^{2}} .
\end{aligned}
$$

There is a genuine curvature singularity at $r=0$, and the boundary of the spacetime is approached as $r \rightarrow \infty$. The Schwarzschild coordinates used in (2.2) also have a coordinate singularity at the horizon $r=1$. This is not a big obstacle, since one can pass to Kruskal coordinates $(T, X)$ which cover the full globally extended spacetime. (The Kruskal coordinate chart is constructed explicitly for this geometry in Appendix A). However, the global extension can be discussed more conveniently for our purposes by using four Schwarzschild coordinate patches (corresponding to the two asymptotic regions (I and III) with $r>1$, plus the regions inside the black hole (II) and the white hole (IV) with $0<r<1$; cf. Fig.1).

These four patches can be embedded in complexified Schwarzschild time,

$$
t=t_{L}+i t_{E}
$$


where $t_{L}$ and $t_{E}$ denote times on the Lorentzian and Euclidean slices respectively. In each coordinate patch, $t$ has a constant imaginary part, as indicated in Fig.1; we will define this to be 0 for the right asymptotic region. Crossing a horizon shifts the imaginary part by $i \beta / 4$. Setting $t=i t_{E}$ produces Euclidean AdS Schwarzschild with a periodic imaginary time coordinate $t_{E}$. The period of $t_{E}$ is $\beta$, the inverse temperature $T$ of the black hole. One can move a point from the boundary of region I to the symmetric point on the boundary of region III in Fig. 1 by rotating by half a period; that is, shifting $t \rightarrow-t-i \beta / 2$. The minus sign accounts for the opposite direction of time in region III. In the geometry (2.2), $\beta=\pi$. The CFT correlators can be defined for complex time and are analytic, so such an extension is natural from the boundary side. The periodicity is just that of finite temperature field theory.

\subsection{Radial null geodesics}

We now turn to a major focus of this paper, the study of geodesics in the geometry (2.2). We consider a radial ( $\Omega$ constant) path, $x=(t(s), r(s))$, where $s$ is an affine parameter. Geodesic paths can be found by extremizing the action

$$
S=\int d s \dot{x}^{2}
$$

where $\dot{x}=\frac{d x}{d s}$. We can then find a unit speed parametrization by requiring $\dot{x}^{2}=0,+1,-1$ for null, spacelike, and timelike geodesics, respectively.

Due to the symmetry described by the Killing field $\left(\frac{\partial}{\partial t}\right)$, there exists a conserved quantity $E$ along each geodesic,

$$
E=\dot{t} f(r)
$$

To map out causal structure we first consider null geodesics. In a spherically symmetric spacetime, radial null rays will always be 45-degree lines in the Penrose diagram. Here $\dot{x}^{2}=-f(r) \dot{t}^{2}+\frac{\dot{r}^{2}}{f(r)}=0$, which when combined with (2.5) becomes

$$
\dot{r}^{2}=E^{2}
$$

Let us consider the coordinate time $t(r)$ along an ingoing radial null geodesic which starts from the boundary $r=\infty$ at $t=t_{0}$. From (2.5) and (2.6) we have that

$$
t(r)=\int \frac{\dot{t}}{\dot{r}^{\prime}} d r^{\prime}=t_{0}+\int_{r}^{\infty} \frac{d r^{\prime}}{f\left(r^{\prime}\right)} .
$$

For geodesics which cross the horizon, integrating over the pole at $r=1$ gives a constant imaginary part $-i \frac{\pi}{4}$, so that for $r<1$,

$$
t(r)=t_{0}-\frac{1}{2}\left[\tan ^{-1} r-\frac{\pi}{2}-\tanh ^{-1} r\right]-i \frac{\pi}{4} .
$$




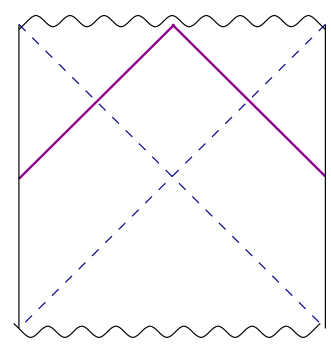

(a)

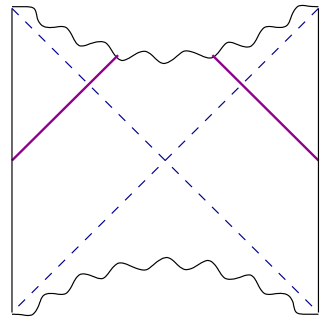

(b)

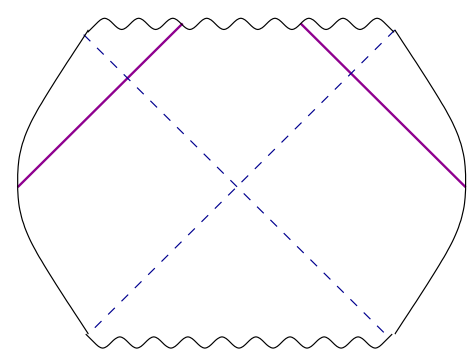

(c)

Fig. 2: Behaviour of radial null geodesics suggests the shape of the Penrose diagram (wavy lines represent the singularities, dashed lines the horizons, vertical lines/curves the boundaries, and the solid 45 degree lines the radial null geodesics): a) if geodesics meet at the singularity, the diagram can be drawn as a square; if they don't meet, b) with straight boundaries, the singularities are bowed in, or c) with straight singularities, the boundaries must be bowed out.

The the last term is simply $i \beta / 4$, where again $\beta=1 / T=\pi$ is the Bekenstein-Hawking temperature (cf. Fig.1) for this geometry.

This result implies that a null geodesic which starts at the boundary at $t_{0}=0$ reaches the singularity $r=0$ at $t(0)=\frac{\pi}{4}(1-i)$. Since the real part of $t(0)$ is positive, the geodesic hits the singularity off-center in the Penrose diagram; in fact it hits closer to the right boundary, where it originated.

This immediately suggests the nature of the Penrose diagram, as illustrated in Fig.2. If the Penrose diagram were a square, as in Fig.2a, then radial null geodesics sent off from the boundary at $t=0$ (defined as the horizontal line of symmetry of the Penrose diagram) would have to meet at the same point on the singularity. On the other hand, if the geodesics don't meet at or before reaching the singularity, then the singularity on the Penrose diagram must be drawn bowed in, assuming we draw the boundaries straight, as in Fig.2b. Alternately, if the singularity were to be represented by a straight line, the boundaries would have to be bowed out, as in Fig.2c, in order to reproduce the calculated behaviour of the geodesics. Note that the Penrose diagrams sketched in Fig.2b and Fig.2c are equivalent, since we can use a conformal transformation to straighten out the singularities of Fig.2b, but at the expense of bowing out the boundaries. This will be discussed in greater detail in Appendix B, where the Penrose diagram for this spacetime is constructed explicitly. Such Penrose diagrams have previously been discussed in [46] .

Conversely, we can ask at what time does a null geodesic have to start from the boundary in order to reach the singularity at $\operatorname{Re}[t(r=0)]=0$, i.e. in the "middle" of the 


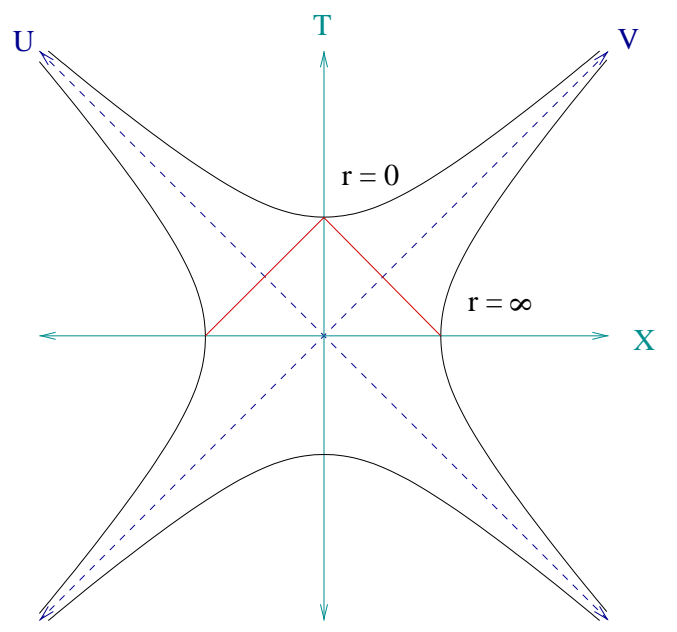

(a)

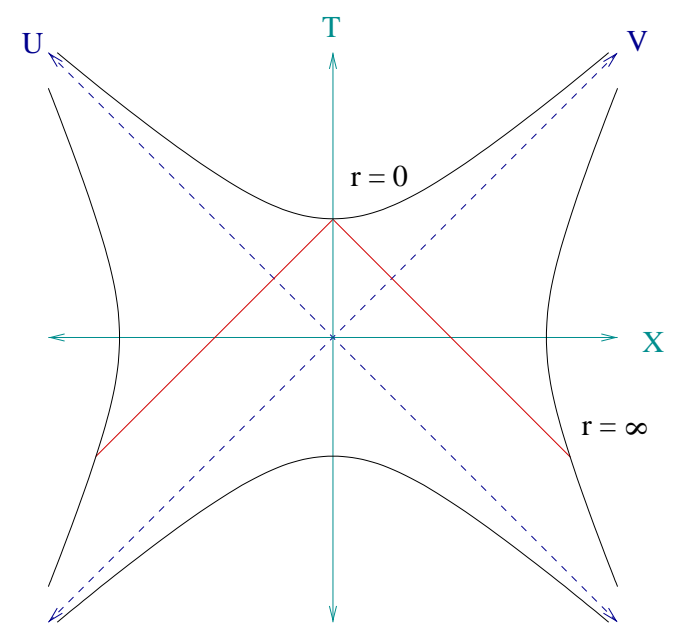

(b)

Fig. 3: Radial null geodesics on Kruskal diagram, a) for $d=3$, and b) for $d>3$ (radial null lines lie at 45 degrees, the horizons are represented by dashed lines, and the boundaries and singularities by the solid hyperbolas). In order for radial null geodesics to meet at the singularity at $X=0$, they have to start at a) $t=0$ for the $d=3$ case, and b) $t=-t_{c}<0$ for the $d>3$ case.

singularity. This will play an important role later. Denoting such a time by $t_{c}$, we see that

$$
t_{c}=-\frac{\pi}{4}=-\frac{\beta}{4}
$$

In general dimension $d$, one finds that $t_{c}=-\pi\left((d-1) \tan \frac{\pi}{(d-1)}\right)^{-1}$, which vanishes only for $d=3$. Thus we see that, with the exception of the $d=3$ BTZ black hole, all singularities in the Penrose diagrams of AdS Schwarzschild are bowed in as in Fig.2b.

In Kruskal coordinates $(T, X)$, the future and past singularities of the black hole are the hyperbolas $T^{2}-X^{2}=1$, while the AdS boundaries are given by $T^{2}-X^{2}=$ $-e^{\pi}$. (By contrast, in the $d=3$ BTZ geometry, the singularities are at $T^{2}-X^{2}=1$ and the boundaries are at $T^{2}-X^{2}=-1$.) The curved nature of the boundary of the Penrose diagram is reflected in these coordinates by the asymmetry in the radii of the two hyperbolas. This asymmetry allows a null geodesic starting at $t_{c}$ to "bounce off" the singularity. This is illustrated in Fig.3, where the $d=3$ case (Fig.3a) is contrasted with the higher-dimensional case (Fig.3b). In Appendices A and B we explain this in further detail, and construct the Penrose diagram explicitly. (The latter will be used in the next subsection to produce the Penrose diagrams in Fig.5.) These issues are also discussed in [46]. 


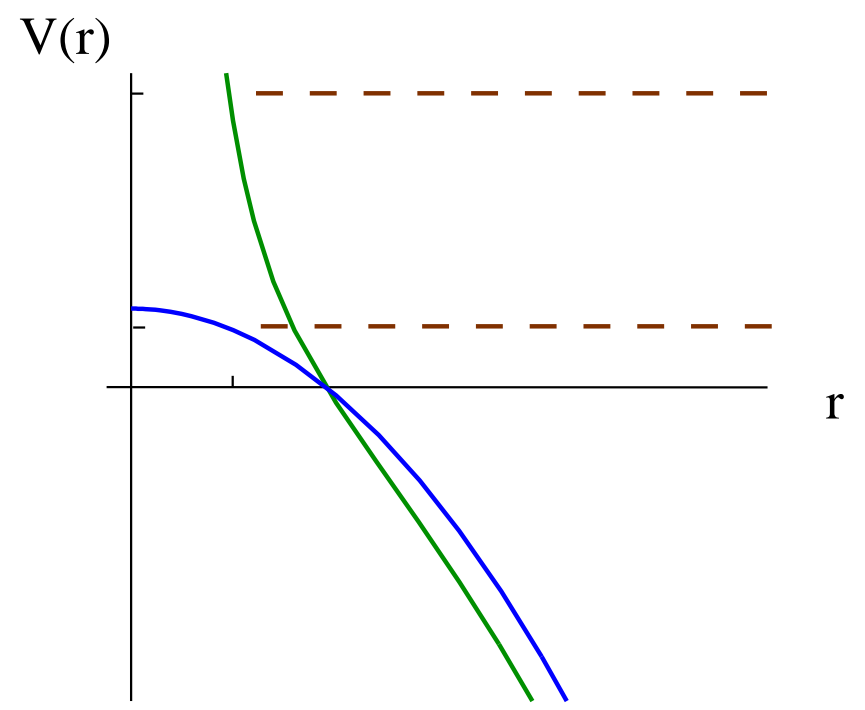

Fig. 4: The "potential energy" in the mechanics problem for spacelike geodesics in AdS Schwarzschild in $d=3$ and $d=5$. The potential diverges at $r=0$ for all $d>3$.

\subsection{Radial spacelike geodesics}

Let us now turn from the qualitative structure of the Penrose diagram to quantities which we can probe from the CFT side: in particular, the proper length along spacelike geodesics. In this subsection, we will consider purely radial spacelike geodesics.3 The spacelike geodesics satisfy $\dot{x}^{2}=-f(r) \dot{t}^{2}+\frac{\dot{r}^{2}}{f(r)}=1$, giving

$$
E=\dot{t} f(r), \quad \dot{r}^{2}-f(r)=E^{2} .
$$

Here $E$, as before, is a conserved quantity resulting from the time translation symmetry of the geometry. We can think of it as the total energy of a particle moving in a potential $V(r)=-f(r)$ (where the minus sign is due to the fact that we are considering spacelike geodesics).

In Fig.4, we plot the potential $V(r)$ for $d=3$ and $d=5$ AdS Schwarzschild. In the BTZ case, the energy required to reach $r=0$ is finite. As a result, the proper time along the geodesic is large. By contrast, in the higher dimensional examples the potential diverges at the singularity, so that large $E$ is required to approach $r=0$. At large $E$ the particle is moving fast and so very little proper time is covered. The result as $E \rightarrow \infty$ is a geodesic that is null everywhere except in a vanishingly small region very near the singularity where it "bounces" off. As explained above, this occurs at $t_{0}=t_{c}$.

3 For completeness, we present in Appendix C an analogous treatment for spacelike geodesics carrying angular momentum. For the remainder of the paper, however, we need only the results about geodesics with zero angular momentum. 


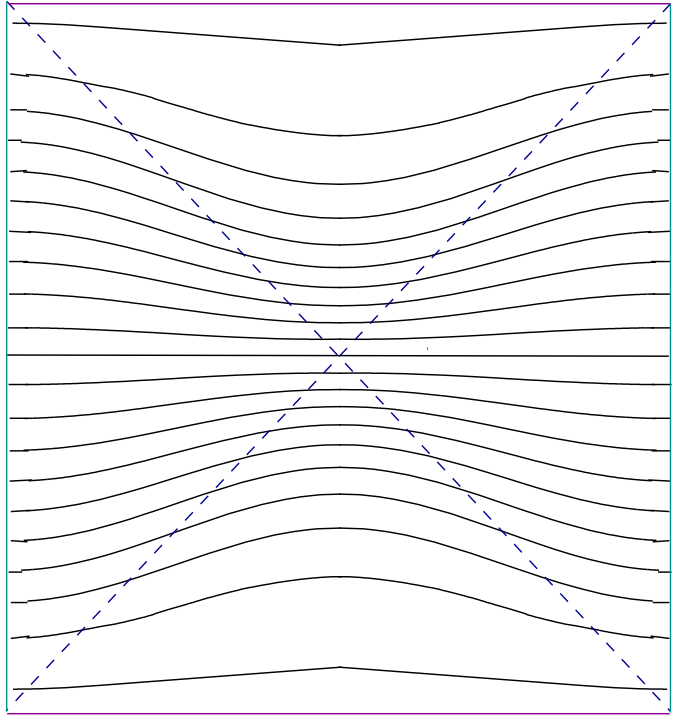

(a)

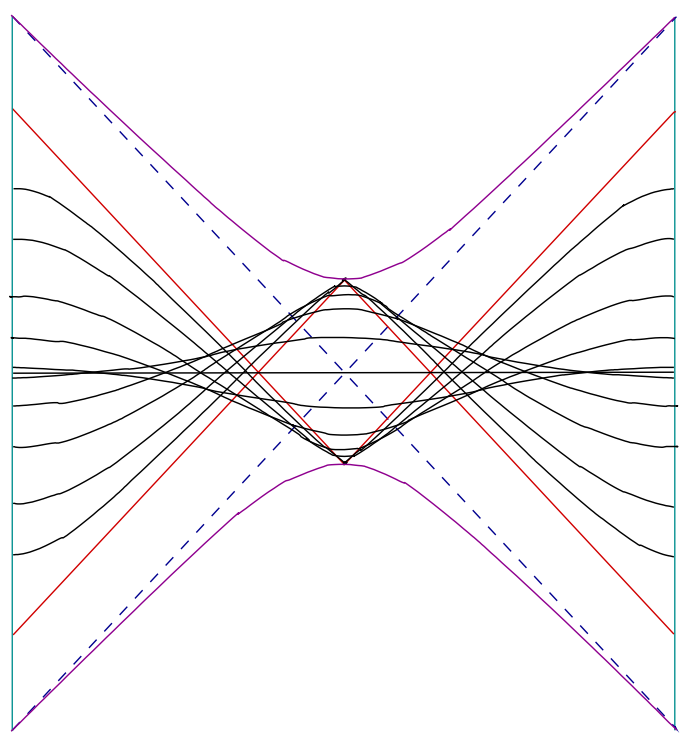

(b)

Fig. 5: Symmetric spacelike radial geodesics of the AdS Schwarzschild black hole, plotted on the corresponding Penrose diagrams. a) $d=3$ : Because only finite $E$ is required to come arbitrarily close to the singularity, none of these geodesics is nearly null. b) $d>3$ : the spacelike geodesics converge to a large $E$ almost null geodesic which comes arbitrarily close to the singularity.

The behaviour of radial spacelike symmetric geodesics (i.e. those which reach their minimum value of $r$ at $t=\frac{i \beta}{4}$ ) in $d=3$ and $d=5$ is plotted on the corresponding Penrose diagrams in Fig.5. As explained above, in the $d=3$ BTZ case (Fig.5a), the geodesics do not show any striking feature as the starting time $t_{0}$ is varied. On the other hand, in higher dimensions such as $d=5$ (Fig.5b), spacelike symmetric geodesics exist only for a finite range of starting times, $t_{0} \in\left(-t_{c}, t_{c}\right)$. For starting times outside this range, no such geodesics exist. This is in accord with the expectation that the genuine curvature singularity of the higher dimensional black holes has a pronounced effect on geodesics in its vicinity, as opposed to the $d=3 \mathrm{BTZ}$ orbifold singularity.

Let us first consider the $E=0$ case. From (2.10), we see that $\dot{t}=0 ; t(s)=0$ is the only consistent solution. We have that $\dot{r}^{2}=f(r)$. Such a geodesic crosses the Penrose diagram in the middle, so that it goes from $r=\infty$ at one boundary, down to the horizon $r=1$, and back out to $r=\infty$ at the other boundary. Since it never penetrates inside the horizon, this geodesic can equally well be embedded in Euclidean AdS Schwarzschild. It is the geodesic that crosses over the tip of the cigar to the antipodal point $t_{E}=\beta / 2$ on the Euclidean time circle (Fig. 8 ). 
The proper length along such a geodesic is then given by

$$
\mathcal{L}=2 \int_{1}^{r_{\max }} \frac{d r}{\dot{r}}=2 \int_{1}^{r_{\max }} \frac{d r}{\sqrt{f(r)}}
$$

where $r_{\max } \rightarrow \infty$ is the large $r$ regulator standard in AdS/CFT [26]. Integrating this explicitly, we have

$$
\mathcal{L}=\ln \left(r_{\max }^{2}+\sqrt{r_{\max }^{4}-1}\right) \approx 2 \ln r_{\max }+\ln 2+\mathcal{O}\left(r_{\text {max }}^{-4}\right)
$$

so that taking $r_{\max } \rightarrow \infty$ and subtracting off the universal logarithmically divergent piece, we obtain the regularized proper length

$$
\mathcal{L}_{\text {reg }}=\ln 2
$$

In the future we will drop the subscript on $\mathcal{L}_{\text {reg }}$.

Let us now consider what happens as we increase the energy $E$. From (2.10) we see that the geodesic now penetrates a finite distance inside the horizon. Specifically, let $r_{i}$ denote the smallest value of the radial coordinate reached along such a geodesic. This corresponds to the classical turning point of the particle motion, which is obtained by solving

$$
E^{2}+f\left(r_{i}\right)=0 .
$$

Since $f(r)$ is positive outside the horizon and negative inside, $r_{i} \leq 1$. For very high energy $E \gg 1$, the geodesic comes very close to the singularity, $r_{i} \ll 1$, so that $f(r) \sim-\frac{1}{r^{2}}$, which means that $r_{i} \sim \frac{1}{E}$.

In general, spacelike geodesics with arbitrary $E$ and starting time $t_{0}$ will not be symmetric. We can specialize to this case, however, and obtain all others by $t$ translation. For a given $E$, there exists a starting time $t_{0}$ on the boundary in region I for which the geodesic is symmetric. In particular, for a symmetric geodesic the turning point $r=r_{i}$ has to occur at $\operatorname{Re}\left[t\left(r_{i}\right)\right]=0$. The turning point must be in region II so $\operatorname{Im}\left[t\left(r_{i}\right)\right]=-\beta / 4$. The geodesic ends in region III with coordinate $t=-t_{0}-i \beta / 2$. As mentioned before, the real part has a minus sign because the Schwarzschild coordinate time runs in the opposite directions in regions I and III. The geodesic equations give

$$
t_{0}-t\left(r_{i}\right)=t_{0}+i \beta / 4=-\int_{r_{i}}^{\infty} \frac{\dot{t}}{\dot{r}} d r=-\int_{r_{i}}^{\infty} \frac{E}{f(r) \sqrt{E^{2}+f(r)}} d r .
$$

For $E=0$, we recover $t_{0}=0$. One can also check that as $E \rightarrow \infty, t_{0} \rightarrow t_{c}$; and in fact, for large energies one can see from examining the integral that

$$
\left(t_{0}-t_{c}\right) \sim \frac{1}{E}
$$


For each such geodesic there exists a mirror one obtained by taking $t \rightarrow-t, E \rightarrow-E$.

The proper length, analogous to (2.11), along a symmetric spacelike geodesic originating at $r_{\max }$, going down to $r_{i}$, and then back out to $r_{\max }$ in the other asymptotic region, is

$$
\mathcal{L}=2 \int_{r_{i}}^{r_{\max }} \frac{d r}{\sqrt{E^{2}+f(r)}} .
$$

Upon evaluating and regularizing this integral, we obtain

$$
\mathcal{L}=\ln \left(\frac{2}{\sqrt{E^{4} / 4+1}}\right) .
$$

As $E$ goes to infinity the geodesic looks more and more null, and so at any fixed radial cutoff its proper length goes to zero. This is apparent already from Fig.5b, where we can see explicitly that symmetric spacelike geodesics in the $E \rightarrow \infty$ limit approximate the null geodesic which bounces off the singularity. The regularized length along an $E \rightarrow \infty$ geodesic (which is roughly the difference between its proper length and that of the $E=0$ geodesic) goes to negative infinity at $t_{c}$,

$$
\mathcal{L} \sim 2 \ln \left(t-t_{c}\right) .
$$

We will make use of this result in the next section, after we discuss the correlation functions.

\section{Correlation functions}

According to the AdS/CFT correspondence, AdS Schwarzschild in $d=5$ is dual to $\mathcal{N}=4$ SYM theory at finite temperature. As before, we will consider the large black hole limit, so the CFT effectively lives in infinite volume. Standard CFT finite temperature boundary correlators are dual to bulk correlation functions with insertions all in one asymptotic region (e.g., region I). There are other boundary correlators one might want to study. For example, one could put an operator on the boundary of region I, and another on the boundary of region III [30,31] . We have discussed the geodesics connecting such points. In the field theory, such correlators have a natural representation in the thermofield double or real-time description of finite temperature field theory [44].

In the real-time formalism, one takes the tensor product of two copies of the original field theory labeled by 1,2 . The two copies are decoupled, and the total Hamiltonian is

$$
H_{t f} \equiv H \otimes I-I \otimes H^{*},
$$

where $H$ is the Hamiltonian for the original theory. We now construct the entangled state

$$
|\psi\rangle=\frac{1}{Z^{\frac{1}{2}}} \sum_{i} e^{-\frac{1}{2} \beta E_{i}}\left|E_{i}, E_{i}\right\rangle,
$$


where $\left|E_{i}, E_{j}\right\rangle=\left|E_{i}\right\rangle \otimes\left|E_{j}\right\rangle$, and $\left|E_{i}\right\rangle$ are energy eigenstates. The state $|\psi\rangle$ is a particular eigenvector of $H_{t f}$ with eigenvalue zero. Correlations between subsystems 1 and 2 are due to the entanglement in $|\psi\rangle$.

Operators which belong to subsystem 1 have the form $A \otimes I$ (where $I$ is the identity operator), and will be denoted $A_{1}$. Operators associated with subsystem 2 are defined in a similar manner, except with an additional rule of hermitian conjugation:

$$
A_{2} \equiv I \otimes A^{\dagger}
$$

Standard thermal correlation functions may be written as an expectation value:

$$
\left\langle\psi\left|A_{1}(0) B_{1}(t)\right| \psi\right\rangle
$$

As can be easily seen from the form of $|\psi\rangle$, (3.4) is simply the thermal expectation value of $A(0) B(t)$, evaluated in a thermal density matrix at inverse temperature $\beta$. The state counting entropy observed in subsystem 1 is the entropy of entanglement of the state $|\psi\rangle$. In field theory, no physical significance is usually attached to correlators involving both subsystems, but we can certainly define them; for example

$$
\left\langle\psi\left|A_{1}(0) B_{2}(t)\right| \psi\right\rangle .
$$

In the finite temperature AdS/CFT correspondence, (3.5) has a simple interpretation [30]: it corresponds in the bulk to a correlator between operators on the two disconnected boundaries of the spacetime.

It is not hard to see that one can compute (3.5) by analytically continuing (3.4).

$$
\left\langle\psi\left|A_{1}(0) B_{2}(t)\right| \psi\right\rangle=\left\langle\psi\left|A_{1}(0) B_{1}(-t-i \beta / 2)\right| \psi\right\rangle .
$$

This is the analog of using complexified Schwarzschild time as discussed in Section 2. We see that these two sided correlators are just part of the information contained in ordinary thermal correlators, as a function of complex time.

We can now use our knowledge of geodesics in AdS Schwarzschild to study correlation functions in the CFT. The prescription is to determine the 2-point CFT correlators via AdS/CFT from a computation of the bulk propagator. We assume at this point that we have a scalar field of mass $m$ in the bulk, dual to some operator in the CFT whose 2-point function we want to compute. The bulk propagator is given by a sum over paths between two points in the bulk, with each path contributing $e^{-m \mathcal{L}}, \mathcal{L}$ being the proper length of the path, suitably regulated. In the limit of large $m$, this sum will generically be dominated by the shortest geodesic connecting the two points. So the 2-point CFT correlator should go 
like $e^{-m \mathcal{L}}$ (up to $\frac{1}{m}$ corrections), where $\mathcal{L}$ is the regularized length of the shortest geodesic between the two pointst.

In section 3.1 we show that a naive application of this prescription predicts a "light cone" pole in the 2-point opposite side correlator as $t \rightarrow t_{c}$. However, simple arguments in the field theory show that this prediction cannot be correct, at least in the theory defined by analytic continuation from Euclidean space. In Section 3.2 we address this puzzle by examining the branch cut singularity in $\mathcal{L}$ at $t=0$ that is due to a coalescence of geodesics. We first resolve the branch cut by taking $r_{\max }$ large but finite. We find that the unambiguously correct geodesic in Euclidean space bifurcates into two complex geodesics at small Lorentzian time $t_{L}$. These geodesics must determine the correct answer. In section 3.3 we elucidate this further by moving this bifurcation onto the Euclidean section. This involves taking the black hole mass large but finite. Euclidean correlators are unambiguously determined by the shortest geodesics at large $m$, and so we can determine the correct choice of geodesics through the bifurcation. The dominant geodesics continue to the complex ones mentioned above as one goes to the Lorentzian section. However, at large $m$ it is possible to analytically continue correlators around these bifurcations by following geodesics that are no longer dominant. This allows us to find the $t_{c}$ singularity on a secondary sheet of the branched correlation function. This $t_{c}$ singularity gives information about the black hole singularity. It shows that such information is encoded in CFT correlators, albeit in a subtle way involving analytic continuation. In section 3.4 we introduce a simple model of the bifurcation given by an ordinary integral. The properties of this model can be determined precisely and agree with the picture we have obtained. We demonstrate here that all orders fluctuation corrections around a given saddle point can be analytically continued as well. This indicates that additional information about the black hole singularity is also accessible. In section 3.6 we show that our results for the correlator agree with the known values for the quasinormal mode frequencies of the AdS Schwarzschild black hole. This gives independent confirmation of our analysis.

\subsection{A light cone singularity}

Recall from Equation (2.19) that the regularized length of a symmetric geodesic with boundary time $t$ diverges logarithmically at $t_{c}$. Therefore the correlation function behaves like

$$
e^{-m \mathcal{L}} \sim \frac{1}{\left(t-t_{c}\right)^{2 m}} .
$$

We seem to have a pole of order $2 m$ in the two-sided CFT correlator coming from the "almost null" geodesic connecting the boundary points.

\footnotetext{
4 This approach and some of the pitfalls involved are discussed in [29].
} 
This immediately leads to a puzzle. We know that the real-time two-point correlation function, evaluated in a thermal state with inverse temperature $\beta$, is

$$
\begin{array}{r}
\langle\phi(t) \phi(-t)\rangle_{\beta}=\sum_{n} e^{-\beta E_{n}}\left\langle E_{n}\left|e^{-i H t} \phi(0) e^{i H t} e^{i H t} \phi(0) e^{-i H t}\right| E_{n}\right\rangle \\
=\sum_{n, m} e^{-\beta E_{n}-2 i t\left(E_{n}-E_{m}\right)}\left|\phi_{n m}\right|^{2}
\end{array}
$$

where $\phi_{n m} \equiv\left\langle E_{n}|\phi(0)| E_{m}\right\rangle$ and we have assumed $\phi^{\dagger}=\phi$. To get the two sided result, we can either use the real-time formalism and the state $|\psi\rangle$, or continue one of the times from the real value $t$ to $t-i \beta / 2$ :

$$
\left\langle\psi\left|\phi_{2}(t) \phi_{1}(-t)\right| \psi\right\rangle=\langle\phi(t+i \beta / 2) \phi(-t)\rangle_{\beta}=\sum_{n, m} e^{-\frac{\beta}{2}\left(E_{n}+E_{m}\right)-2 i t\left(E_{n}-E_{m}\right)}\left|\phi_{n m}\right|^{2} .
$$

Note that the terms in the sum are real and positive for $t=0$; therefore

$$
\left|\left\langle\phi_{2}(t) \phi_{1}(-t)\right\rangle_{\beta}\right| \leq\left\langle\phi_{2}(0) \phi_{1}(0)\right\rangle_{\beta}
$$

for any Hermitian operator $\phi$. However, $\left\langle\phi_{2}(0) \phi_{1}(0)\right\rangle_{\beta}$ can be computed unambiguously in Euclidean space, using the geodesic approximation. It is certainly finite (since the two points are on opposite sides of the Euclidean cigar). Thus we cannot have a singularity in the opposite side correlator at any time, including $t=t_{c}$.

\subsection{Tracking geodesics}

We begin exploring this puzzle by computing the boundary time as a function of $E$ from the integral (2.15). The computation is detailed in Appendix D; the result is

$$
\begin{aligned}
t & =\frac{1}{2} \ln \left(\frac{\frac{1}{2} E^{2}-E+1}{\sqrt{1+\frac{1}{4} E^{4}}}\right)-\frac{1}{2} i \ln \left(\frac{-\frac{1}{2} E^{2}+i E+1}{\sqrt{1+\frac{1}{4} E^{4}}}\right) \\
\mathcal{L} & =\ln \left(\frac{2}{\sqrt{1+\frac{1}{4} E^{4}}}\right),
\end{aligned}
$$

where formula (2.18) has been repeated for convenience. We have not restricted ourselves to symmetric geodesics here. The time $t$ is the difference between $t_{f}$ and $t_{i}$, the final and initial boundary Schwarzschild times; $t=t_{f}-t_{i}+i \beta / 2$. We have chosen the origin so that $t=0$ describes endpoints in regions I and III at zero time. For symmetric geodesics $t=-2 t_{0}$.

These equations determine $\mathcal{L}(t)$ and hence the large $m$ correlator $\langle\phi \phi\rangle(t)=$ $\exp (-m \mathcal{L}(t))$. They are central to our analysis. 


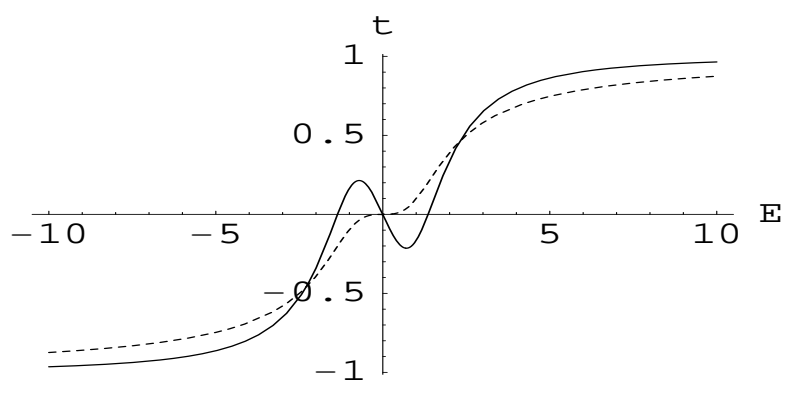

Fig. 6: Plot of $t$ as a functions of $E$, normalized by $t(E \rightarrow \infty)$, for various values of $r_{\max }$ : the solid line has $r_{\max }=2$, while the dashed line represents $r_{\max }=\infty$

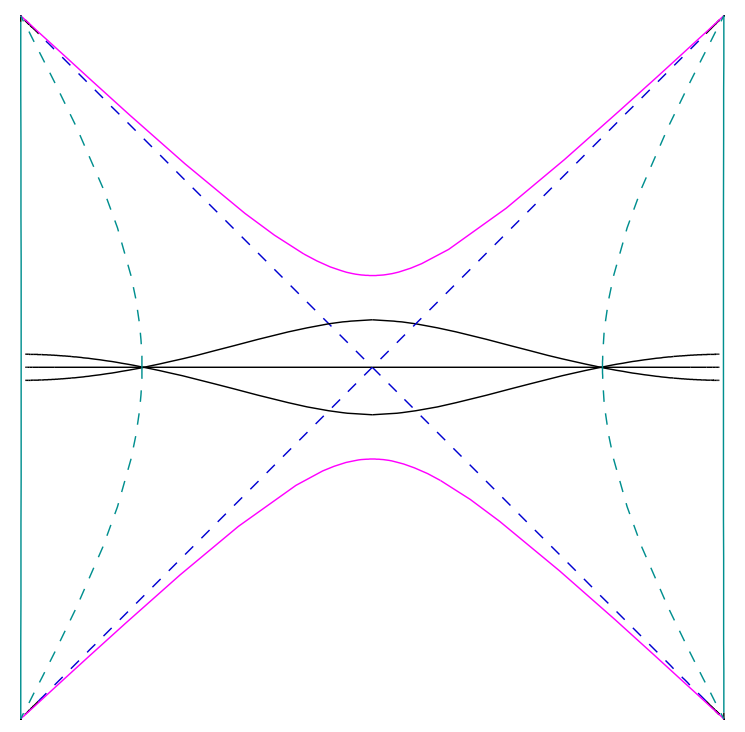

Fig. 7: Three "zero time" geodesics between the two cutoff boundary points $\left(t=0, r=r_{\max }\right)$. The curved dashed lines are $r=r_{\max }$.

Expanding (3.11) around $E=0$ we find

$$
\begin{aligned}
& t \sim E^{3} \\
& \mathcal{L} \sim-E^{4} \\
& \mathcal{L} \sim-t^{\frac{4}{3}} .
\end{aligned}
$$

The branch point at $t=0$ signals novel behavior. There are three branches for $\mathcal{L}$ and hence for the correlator at each $t$. It will turn out the gauge theory chooses a symmetric combination of the two complex branches. The $t_{c}$ singularity lies on the real branch. But knowing the correlator on the complex branch will allow us to study the real branch by analytic continuation. Information about the black hole singularity is encoded in the gauge theory in this manner. The analytic structure of the Riemann surface defined by (3.11) is discussed in Appendix E. 
To track these branches, it is useful to resolve the branch point. To do this we take $r_{\max }$ finite. In the gauge theory this corresponds to a finite UV cutoff. Equations (3.12) become

$$
\begin{aligned}
t & \sim E^{3}-a E \\
\mathcal{L} & \sim-E^{4}-b E^{2} .
\end{aligned}
$$

Here $a, b>0$ are constants that go to zero as $r_{\max } \rightarrow \infty$. At time $t=0$ there are now three real solutions for $E$ and $\mathcal{L}$ (see Fig.6). These correspond to the three geodesics shown in Fig.7.

We can most precisely define gauge theory correlators by evaluating them in Euclidean time and then analytically continuing to general time. To compute the $t=0$ two sided correlator this way we smoothly shift one point of a coincident point Euclidean correlator by a half period, $i \beta / 2$. At $t=0$ we can do the geodesic computation entirely in the Euclidean AdS Schwarzschild geometry. The correct geodesic is clearly the $E=0$ solution of (3.13) which is described in (2.11).

Now let us go to small Lorentzian time by increasing the real part of $t$ by a small amount. The correct geodesic here is certainly the small deformation of the $E=0$ solution with $E \sim t / a$. This corresponds to following the central branch of the cubic curve in Fig.6. But at a time $t \sim a^{3 / 2}$ this root of the cubic annihilates with another root at the local maximum shown in Fig.6. These solutions then become complex. Complex values of $E$ correspond to geodesics in the complexified AdS Schwarzschild spacetime, where both $r$ and $t$ are complex. These can be described as the solutions of the equations of motion of the mechanics problem with complex energy $E$.

Therefore the gauge theory answer for the correlator, defined by continuation from Euclidean space, must become complex after a certain time. The complex branches of (3.11) do not contain the $t_{c}$ singularity, and so this analysis explains its absence in the gauge theory. On the complex branches the second derivative of the real part of $\mathcal{L}(t)$ is negative at $t=0$. In other words, the correlation function computed by following this branch will decrease as we move away from $t=0$, consistent with the general field theory result.

\subsection{Finite mass black hole}

To understand the pattern of geodesics better, we now describe how to move the bifurcation point to Euclidean time. The advantage of studying the Euclidean correlator is that, in the sum over paths representation, the weight factor is always positive and so we can reliably predict that the geodesics with the smallest $\mathcal{L}$ dominate. To move the bifurcation we consider the finite mass AdS Schwarzschild geometry and compute $t(E)$ for its geodesics. The result for small $E$ takes the form (Appendix D): 


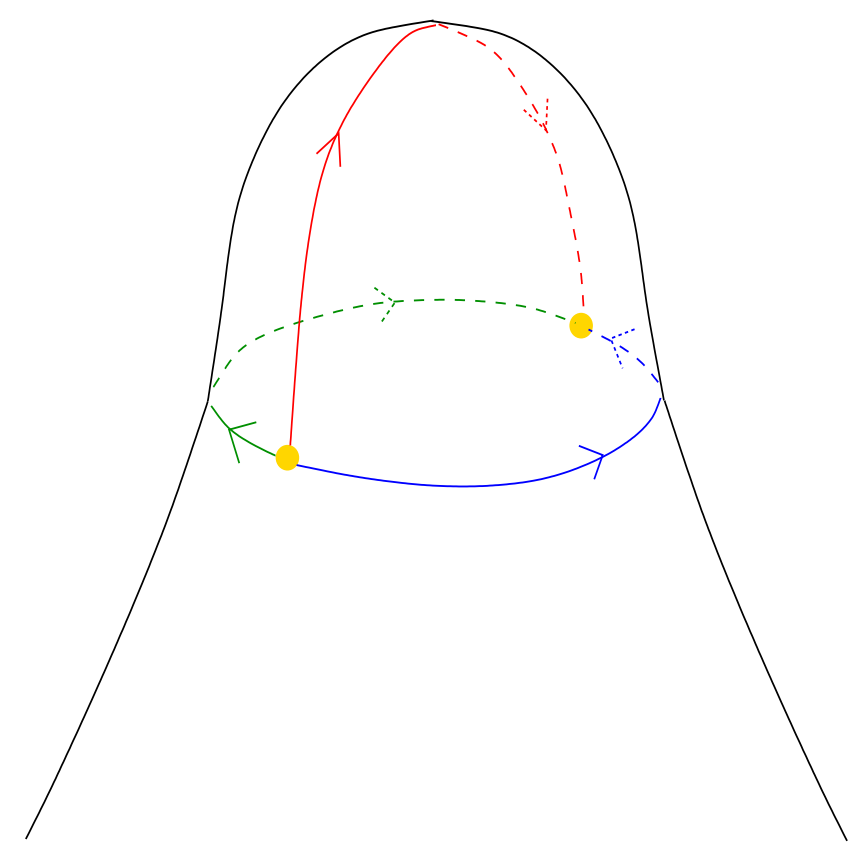

Fig. 8: Geodesics of finite mass Euclidean AdS Schwarzschild with finite $r_{\text {max }}$. The $\tilde{E}=0$ geodesic goes over the top and has larger $\mathcal{L}$ than the two geodesics with nonzero $\tilde{E}$.

$$
\begin{aligned}
t & \sim E^{3}-a E \\
\mathcal{L} & \sim-E^{4}-b E^{2} .
\end{aligned}
$$

Here $a<0$, unlike the finite $r_{\max }$ resolution described by (3.13). The parameters $a$ and $b$ go to zero as the black hole mass becomes infinite. The parameter $b$ is still positive.

If $t=t_{L}+i t_{E}$ is Euclidean $\left(t_{L}=0\right)$, then $E$ is pure imaginary. Let $E=-i \tilde{E}$, with $\tilde{E}$ real. Equation (3.14) becomes

$$
\begin{aligned}
t_{E} & \sim \tilde{E}^{3}-|a| \tilde{E} \\
\mathcal{L} & \sim-\tilde{E}^{4}-|b| \tilde{E}^{2} .
\end{aligned}
$$

This cubic is also described by Fig.6 with the horizontal axis now being $\tilde{E}$. At large $\tilde{E}$, the extension of (3.11) gives $t_{E} \sim \beta / 2$ describing almost coincident Euclidean points. As $\tilde{E}$ is decreased, entering the domain of validity of (3.15), a single solution exists, describing a single geodesic. But at a particular value of $t_{E} \sim|a|^{3 / 2}$ corresponding to the local maximum of the cubic in Fig.6, a pair of geodesic solutions is created. From (3.15) and the sign of $b$ it is clear that these new geodesics have larger $\mathcal{L}$ than the original one, so they are exponentially subdominant. This situation persists until $t_{E}=0$, when the geodesic endpoints are antipodal. Here the three solutions of (3.15) are $\tilde{E}=0, \tilde{E}= \pm \sqrt{|a|}$. The two geodesics with $\tilde{E}= \pm \sqrt{|a|}$ have the same $\mathcal{L}$, which is less than that of the $\tilde{E}=0$ 
geodesic. This situation is illustrated schematically in Fig.8 where we have taken $r_{\max }$ finite for clarity. It is clear from Fig. 8 that the $\tilde{E}=0$ geodesic is a local maximum of proper distance.

At $t_{E}=0$ the correct prescription to compute the correlator is to sum over the two dominant $(\tilde{E} \neq 0)$ geodesics. We then can continue into the Lorentz section by varying $t_{L}$ away from zero. To compute the correlator we follow both of the local minimum geodesics. From (3.14) we see that $E$ for this pair of geodesics will now have to have nonvanishing real and imaginary parts. If we label the two geodesics 1 and 2 , we have $E_{1}=E_{2}^{*}$ and $\mathcal{L}_{1}=\mathcal{L}_{2}^{*}$. The real parts of the proper distances are the same, so the magnitude of their contribution is the same. So our final formula for the two sided correlator as a function of Lorentzian time (or more generally, in a complexified neighborhood of Lorentzian time) is given by

$$
\langle\phi \phi\rangle(t)=e^{-m \mathcal{L}_{1}}+e^{-m \mathcal{L}_{2}} .
$$

Because we have resolved the geodesic bifurcations we have been able to give an unambiguous calculation of this correlator. Taking the mass of the black hole to infinity we see that the correct prescription using (3.11) is to sum over both complex branches with equal weight.

\subsection{A bifurcation model}

As we have seen, at the branch point described in (3.12), the geodesics coalesce. This means that stationary points of the path integral action become arbitrarily close in path space. This indicates that an eigenvalue of the fluctuation operator around such a geodesic must go to zero. There is a soft mode in the fluctuation spectrum around the branch point geodesic. More explicitly, the soft mode corresponds to infinitesimal motions of $E$ away from zero; because $t(E) \sim E^{3}$ this means that the boundary points are fixed under such motions. In the space of paths, one can argue that the coordinate $E$ is a good nonsingular coordinate for this mode near zero. For example, the central point of the path is deflected by a distance proportional to $E$. Since the regularized length behaves like $\mathcal{L} \sim E^{4}$ we see that the fluctuation eigenvalue goes like $d^{2} \mathcal{L} / d E^{2} \sim E^{2} \sim t^{2 / 3}$. This zero mode at $t=0$ will create divergences in the $1 / m$ expansion there.

The fluctuation dynamics near $t=0$ should be dominated by this zero mode. So we should be able to get a reliable picture of the correlator in this region by truncating the path integral to this one fluctuating mode. Of course it will be non-gaussian, but from our knowledge of $\mathcal{L}(E)$ we should be able to determine its dynamics. Representing the nonsingular coordinate for the zero mode $E$ by $x=i E$, and setting $\tau=-i t$ we can model the path integral for $t \sim 0$ by the ordinary integrall

5 This integral is a special case of Pearcey's Integral 48 . 

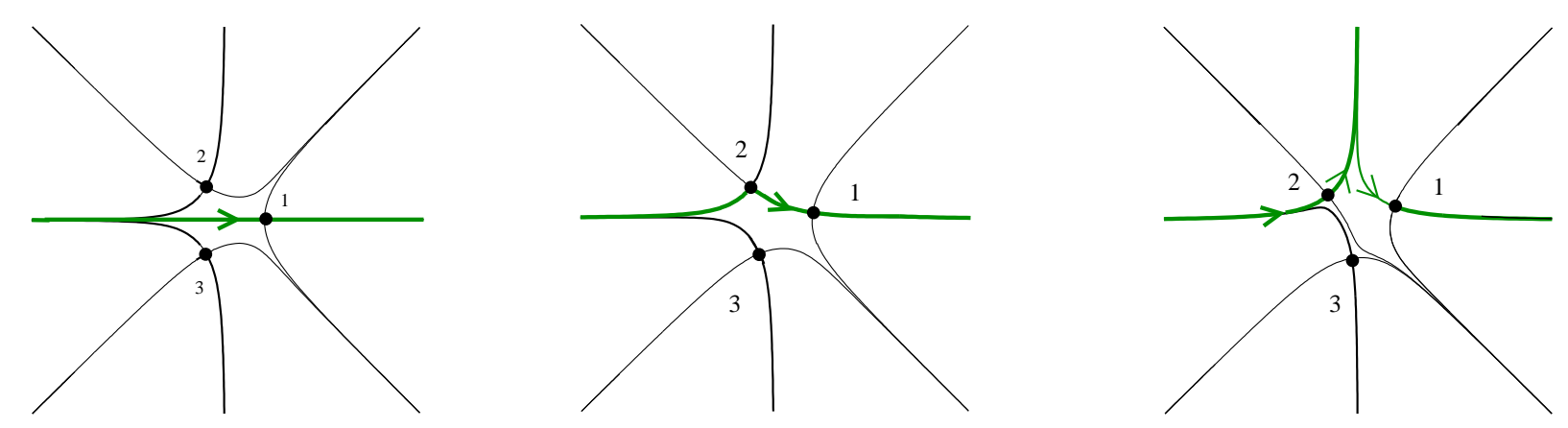

Fig. 9: Integration contours for various values of $t$ : left to right, $\tau=$ $1, e^{i \pi / 8}, e^{i \pi / 5}$. At $\tau=e^{i \pi / 8}$, the integral crosses a Stokes line and picks up another saddle.

$$
I(\tau)=\int_{-\infty}^{\infty} e^{-m V_{\tau}(x)} d x
$$

where the truncated action is given by

$$
V_{\tau}=\frac{1}{4} x^{4}-\tau x
$$

To motivate this choice for $V_{\tau}$, compute its stationary points.

$$
\begin{aligned}
V_{\tau}^{\prime}\left(x_{s}\right) & =x_{s}^{3}-\tau=0 \\
x_{s} & =\tau^{\frac{1}{3}} \\
V\left(x_{s}\right) & =-\frac{3}{4} \tau^{\frac{4}{3}} .
\end{aligned}
$$

This is the analog of (3.12). 6 For $\tau$ real, ( $t$ Euclidean), the integrand in (3.17) is real and positive on the real $x$ integration contour. In such a situation the large $m$ saddle point analysis is straightforward. We can then study deformations of $t$ away from Euclidean.

We now study this model for complex $\tau$. We can still use saddle point approximation for large $m$, but now we have to determine carefully which saddles lie on the integration contour. More precisely, for complex $t$ we must deform the integration contour so that it becomes a steepest descent path for the real part of $V_{\tau}$. Then we can evaluate $I(\tau)$ by doing a saddle point expansion around all the saddles included on the path. A steepest descent path for the real part of $V_{\tau}$ is simply a contour of constant $\operatorname{Im}\left(V_{\tau}\right)$, so they are easy to identify.

6 We will be able to study this integral in sufficient detail that we will not need to add the additional terms $\sim x^{2}$ to $V$ that would resolve the cubic branch point. This can be done though. 


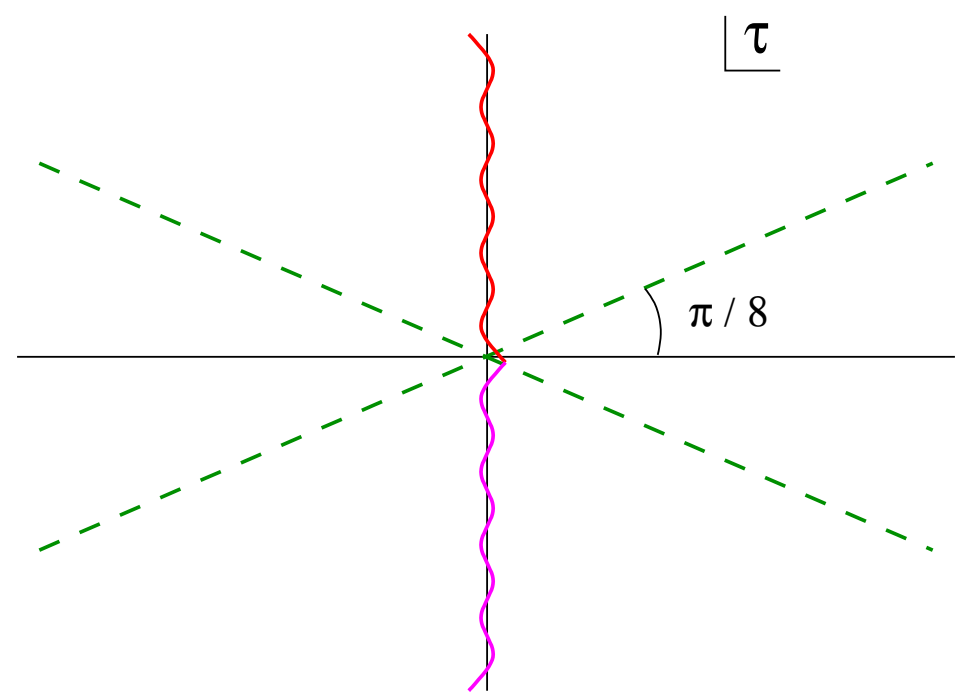

Fig. 10: Positions of the Stokes and anti-Stokes (saddle exchange) lines in the complex $\tau$ plane. The dashed lines are the Stokes lines, where the contour goes from crossing one saddle to two, and therefore picks up a sub-dominant contribution. The two saddles contribute equally on the wavy lines, so that the integral there has a kink (in the large $m$ limit) as a function of $\tau$.

For convenience, we restrict ourselves to $\operatorname{Im}(\tau) \geq 0$, and write

$$
\tau=r e^{3 i \theta}
$$

with $\theta \in\left[0, \frac{2 \pi}{3}\right], r \geq 0$. The critical points are then

$$
x_{k}=r^{\frac{1}{3}} e^{i \theta+\frac{2 \pi i k}{3}}
$$

where $k=0,1,2$. Consider starting with real positive $\tau$, and then rotating $\tau$ counterclockwise into the complex plane. For $3 \theta<\frac{\pi}{8}$, the steepest descent contour contains only the saddle point $x_{0}$. For example, at $\theta=0$, the steepest descent contour is simply the defining contour of the integral: the real axis. At $3 \theta=\frac{\pi}{8}$, however, the imaginary parts of $V_{\tau}$ become equal at $x_{0}$ and $x_{1}$, and the contour picks up the saddle point $x_{1}$ (Fig.9). The line $3 \theta=\pi / 8$ - where the integral picks up a subdominant exponential in the saddle approximation - is known as a Stokes line [49]. This situation persists until $3 \theta=\frac{7}{8} \pi$, at which point the contour loses the point $x_{0}$, and only $x_{1}$ remains (Fig.10).

By computing the real part of $V_{\tau}$, we see that the saddle at $x_{0}$ dominates over $x_{1}$ for $3 \theta<\frac{\pi}{2}$, and vice versa for $3 \theta>\frac{\pi}{2}$. The locus of this exchange of dominance is referred to as an anti-Stokes line. Right on the line both saddles contribute equally. This is the situation for the Lorentzian correlator discussed in the previous subsection.

An interesting phenomenon occurs here. For $\frac{\pi}{4}<3 \theta<\frac{3 \pi}{4}$, the overall dominant saddle is $x_{3}$, which is not on the integration contour, and does not contribute to the 
integral in this approximation. This is similar to the phenomenon we encountered in the AdS Schwarzschild correlator, where the dominant branch (the $t_{c}$ geodesic) does not contribute. That we have a situation in which a dominant saddle does not contribute to the contour integral may at first seem surprising. In fact, it is easy to see that no matter how one tries to deform the contour so as to force it to go over this dominant saddle in a meaningful direction, one will pick up a larger extremum elsewhere on the contour. This extremum is of course only an extremum for $V_{\tau}$ restricted to the contour. It is not a critical point of $V_{\tau}$ as a function of complex $\tau$. However we do not have an analogous understanding of why the $t_{c}$ geodesic does not contribute to the Lorentzian functional integral. There must be other large contributions that cancel it.

For $m \rightarrow \infty$ subdominant saddles make no contribution to this analytic function. The true $m \rightarrow \infty$ correlation function 7 is a set of analytic regions, joined together in a non-smooth way at anti-Stokes lines when saddles exchange dominance. This is somewhat analogous to a first order transition in statistical mechanics. Computing the correlation function in one region and analytically continuing ignores these boundary lines, and is somewhat like continuing into a metastable phase. This analogy is imperfect, though. The bifurcation model illustrates that the $t_{c}$ saddle does not give a contribution like $e^{-m \mathcal{L}}$ to the path integral as would a metastable phase.

Now consider $I(\tau)$ for large but finite $m$. It is clear from the defining integral (3.17) that $I$ is an entire function of $\tau$. The $m=\infty$ branch point at $\tau=0$ is smoothed out. The integral has an everywhere convergent power series and in a small disk $|\tau| \ll 1 / m^{\frac{3}{4}}$, $I$ is roughly constant. When $|\tau| \sim 1$ the saddle point approximation is very accurate for large $m$. Along the anti-Stokes (saddle exchange) line, the vertical axis in Fig.10, there is a rapid but smooth transition from one saddle behavior to the other. The width of this transition is order $1 / m$. So there are two half plane regions where the large $m$ saddle point behavior is very accurate, a thin joining region along the anti-Stokes line and a small roughly constant disk near the origin. These features smooth out the branch cut and so prevent us from following subdominant saddles by analytic continuation.

A concrete method for performing analytic continuation is by computing power series coefficients around a point and then continuing the series variable. If we expand, for example, around a point away from the anti-Stokes (saddle exchange) line at large but finite $m$ the contamination of the subdominant saddle point contribution that prevents continuation past the point of saddle exchange is of order $e^{-m}$. Near the exchange line we can model the correction more accurately as $e^{-m(1-\tau)}$ where the exchange line is at $\tau=1$. The power series expansion of this correction goes like $e^{-m} \sum(m \tau)^{k} / k$ ! . For fixed $k$ the corrections are order $e^{-m}$ but for $k \sim m$ the corrections are order one. So the

7 More precisely, we should consider the function $L(\tau)=-\frac{1}{m} \ln I(\tau)$ which has a smooth large $m$ limit. 
practical question about how large an $m$ is required to be effectively infinite is equivalent to how many terms of a power series are required to accurately represent the infinite $m$ function. The functions we are interested in have smooth large $m$ limits so the number of terms required is $m$ independent. This means that accurate analytic continuation can be done with results at large but finite $m$. Examples of this procedure for studying the $t_{c}$ singularity are discussed in Appendix F.

There is an asymptotic series of $1 / m$ corrections to $I(\tau)$ that can be computed by Feynman diagram techniques. Because of the soft mode, each of these terms is divergent at $\tau=0$ and has the structure $1 /\left(m \tau^{\frac{4}{3}}\right)^{k}$. So each term lives on the same three sheeted Riemann surface as the leading answer. If we compute a given term around a dominant saddle and then analytically continue to a region where that saddle is no longer dominant we find the same answer we would get by perturbing around the subdominant saddle using a different integration contour. So the fluctuations we compute around the dominant saddle give us information about fluctuations around the subdominant one. We can clearly do the same thing with corrections in powers of $a$. We will use generalizations of this idea to argue that we can study stringy and quantum effects around the bouncing geodesic.

\subsection{The $t_{c}$ singularity}

We now have a reliable calculation of the boundary correlator at large $m$ in the classical supergravity approximation as a function of complex boundary time $t$. The result is contained in Equation (3.11), with additional information about which geodesics dominate. For Lorentzian time $t=t_{L}$ we sum over both complex solutions of (3.11) with equal weight. The complex branches of (3.11) are free of additional singularities for all finite $t_{L}$. But the functions $\mathcal{L}_{i}(t)$ are analytic in $t$. Given their values along any small segment of $t$ it is possible to analytically continue them over the entire Riemann surface described by (3.11). The $t_{c}$ singularity can be found by such an analytic continuation from the dominant branch selected by the boundary CFT. In the discussion above of the bifurcation model we started from Euclidean $t$. Here let us give the argument starting from the Lorentzian $t$. Consider small $t$ and hence use the expansion (3.12), which gives us the relation $E \sim t^{\frac{1}{3}}$. As we have argued previously, for small Lorentzian $t$ the boundary CFT selects the branch $E=e^{\frac{2 \pi i}{3}}\left|t^{\frac{1}{3}}\right|$. (We suppress the other complex branch which behaves similarly.) If we denote $t=e^{i \alpha} t_{L}$ then we reach the branch with real $t$ and real $E$ (and hence real $\mathcal{L}$ ) by taking $\alpha=\pi$. On this branch there is a singularity at $|t|=t_{c}$, due to the logarithmically diverging $\mathcal{L}$ of the analytically continued geodesic, which "bounces" off the singularity. This $t_{c}$ singularity clearly reflects aspects of the black hole singularity. So we see that information about the black hole singularity is encoded in the boundary correlators, albeit in a subtle way involving analytic continuation. Despite its subtlety, analytic continuation

can be computationally effective. In Appendix F we show how a modest number of terms 


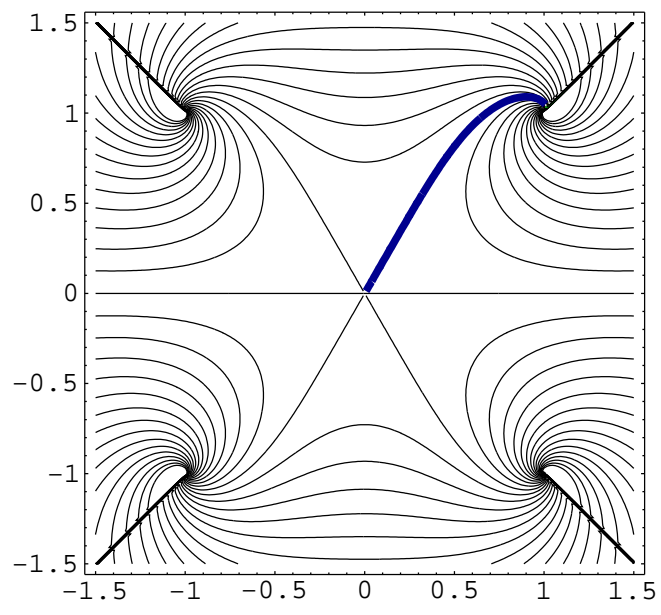

a)

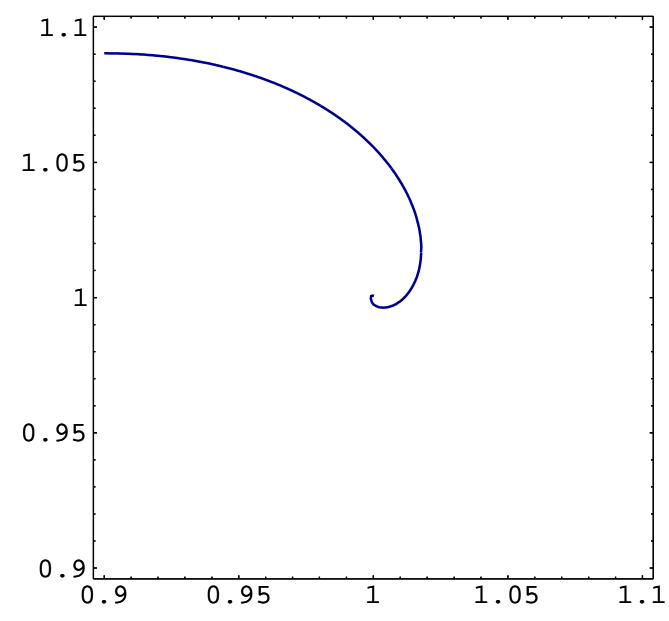

b)

Fig. 11: Contours of constant imaginary part for the function $t(E)$. There are three $\operatorname{Im}(t)=0$ contours, which cross at the origin. The $t_{c}$ branch is the real axis. The other two give us the non- $t_{c}$ branches of the correlator. Pane b) is a magnified view of the singularity at $1+i$, showing only the $\operatorname{Im}(t)=0$ contour.

in a power series expansion of $\mathcal{L}$ on a dominant branch is enough to extract precision information about the $t_{c}$ singularity.

\subsection{Quasinormal modes}

We can use the geodesic expression for the correlator derived above to compute the quasinormal modes of the AdS Schwarzschild black hole, in the large $m$ limit. These agree with the known results [50], providing a check of our calculation. 8

To compute quasinormal modes we need the large $t$ asymptotics of the correlator. We first note that along either complex branch $E(t)$ must go to one of the fourth roots of -4 as $t$ goes to infinity. Specifically, let $\alpha_{1}$ and $\alpha_{2}$ be $-1-i$ and $1-i$ respectively; we can rewrite formula 3.11$)$ as

$$
t=\frac{1}{4} \ln \left[\frac{\left(E-\alpha_{1}\right)\left(E-\overline{\alpha_{1}}\right)}{\left(E-\alpha_{2}\right)\left(E-\overline{\alpha_{2}}\right)}\right]-\frac{1}{4} i \ln \left[\frac{-\left(E-\alpha_{1}\right)\left(E-\alpha_{2}\right)}{\left(E-\overline{\alpha_{1}}\right)\left(E-\overline{\alpha_{2}}\right)}\right]
$$

We can expand around these various roots; for example, expanding $E$ around $\alpha_{2}$ gives $t=c-\left(\alpha_{2} / 4\right) \ln \left(E-\alpha_{2}\right)$, where $c$ is a complex constant. Writing $E-\alpha_{2}=r e^{i \theta}$ and requiring that $t$ be real gives

$$
\theta=\ln r+c^{\prime}
$$

8 We thank Chris Herzog for pointing out a mistake in a previous version of this section. 
where $c^{\prime}$ is a real constant. This demonstrates that the complex branches (where $t$ goes to real infinity) are spirals in the complex $E$ plane, converging on one of the roots (see Fig.11). Coupled with the expansion $\mathcal{L} \sim-\frac{1}{2} \ln (E-\alpha)$ around any of the four roots, we have that as $t$ goes to infinity, $t \sim \frac{1}{2}(1+i) \mathcal{L}$ along one of the complex branches and $t \sim \frac{1}{2}(1-i) \mathcal{L}$ along the other. This gives the leading large $m$ behavior of $e^{-m \mathcal{L}_{1}}+e^{-m \mathcal{L}_{2}}=$ $e^{-m(1+i) t}+e^{-m(1-i) t}$. This reproduces the first large $m$ quasinormal modes of this black hole found in [50]. Note that conventions differ between [50] and this paper.

To go beyond the first quasinormal mode, we argue as follows. Suppose we are on the complex branch that goes to $\alpha_{2}$ as $t$ goes to infinity. From (2.18) we then see that $e^{-\mathcal{L}}=\left(E-\alpha_{2}\right)^{\frac{1}{2}} \tilde{f}(E)$, where $\tilde{f}$ is analytic. We can invert and obtain

$$
E=\alpha_{2}+e^{-2 \mathcal{L}} f\left(e^{-2 \mathcal{L}}\right)
$$

with $f(0)=1$ and $f$ analytic. Substituting, we obtain $t=\frac{1}{2}(1+i) \mathcal{L}+\tilde{g}\left(e^{-2 \mathcal{L}}\right)$, with $\tilde{g}$ analytic. Inverting once more we obtain

$$
\mathcal{L}=(1-i) t+g\left(e^{-2(1-i) t}\right) .
$$

Now, the correlator is

$$
\langle\phi \phi\rangle(t)=e^{-m\left(\mathcal{L}(t)+(1 / m) f_{1}(t)+\cdots\right)}
$$

where $f_{1}$ is the first $1 / m$ correction. By extensivity and locality, we know that $f_{1}(t)=$ $b_{0} t+h(t)$ where $b_{0}$ is a constant of order one determined by the fluctuation determinant around the geodesic (which we have not calculated) and $h(t)$ is exponentially small in $t$ because the world line path integral is massive in AdS Schwarzschild. Expanding gives a series

$$
\langle\phi \phi\rangle(t) \sim \sum_{n=0}^{\infty} c_{n} e^{-\left(m+b_{0}+2 n\right)(1-i) t}+\text { c.c. }
$$

This yields values for the quasinormal modes

$$
\omega_{n}=\left(m+b_{0}+2 n\right)(1 \pm i) \quad n=0,1,2 \ldots .
$$

These values agree with the large $m$ quasinormal modes calculated in [50] if $b_{0}=-1$. The results in [50] were obtained by a direct study of the radial equation for the AdS Schwarzschild geometry, the Heun differential equation. In some way the geodesic computations summarized in (3.11) give a WKB solution to the Heun equation.

\section{Beyond the geodesic approximation}

In the previous section we studied the massive scalar correlator in the limit $m \rightarrow \infty$ where the geodesic approximation applies. We worked in the classical supergravity regime, $\alpha^{\prime}=l_{s}^{2}=0$ and $g_{s}=0$. In this section we will discuss the more general situation. 


\subsection{Finite mass}

The scalar propagator has a path integral representation

$$
\left\langle\phi\left(\xi_{1}\right) \phi\left(\xi_{2}\right)\right\rangle=\int_{0}^{\infty} d T \int D x(s) \exp \left(-i m / 2 \int_{0}^{T} d s\left(\dot{x}^{2}+1\right)\right) .
$$

Here $\xi_{i}$ are points on the boundary and the integral is over paths in the bulk that connect these two points. For the radially separated points discussed in the previous sections $\left\langle\phi\left(\xi_{1}\right) \phi\left(\xi_{2}\right)\right\rangle=\langle\phi \phi\rangle(t)$. The mass $m$ acts like $1 / \hbar$, a saddle point parameter. Taking $m$ finite means all paths are explored in the functional integral, not just the dominant saddles. So one of the key steps of the previous section-following a saddle point into a region where it is no longer dominant-will be problematic when $m$ is finite. More explicitly, as discussed in section 6.5, the function $L(m, t)=-\frac{1}{m} \log \langle\phi \phi\rangle(t)$ defines a piecewise analytic function in the limit $m \rightarrow \infty$ that can be continued past the anti-Stokes line where saddle point dominance is exchanged, but at finite $m$ there is no clear way to follow the subdominant saddle past this line. Operationally, the "contamination" due to subdominant saddles must be controllably small to perform such analytic continuation.

The coefficients of the $1 / m$ expansion can be continued unambiguously and do contain significant physical information. From (4.1) we see that this expansion is a small fluctuation or heat kernel expansion. There are two places where divergences are expected in the coefficients of $(1 / m)^{k}$. The first is $t=0$, where multiple geodesics coincide. As discussed in section 3.3 we expect a soft mode in the fluctuations around the geodesic here. This will cause a divergence in the $1 / m$ expansion. The terms in the $1 / m$ expansion live on the same three sheeted Riemann surface as $\mathcal{L}$. As discussed above, continuing these coefficients gives the small fluctuation expansion around the analytically continued saddle.

The second place we expect divergences is on the bouncing geodesic for $t \sim t_{c}$. Here the divergence is not due to a soft mode but to large nonlinearities present because the curvature is large near the singularity. We can expand the heat kernel (4.1) in a short time expansion

$$
\left\langle\phi\left(\eta_{1}\right) \phi\left(\eta_{2}\right)\right\rangle \sim \sum_{k=0}^{\infty} c_{k} R^{(k)}\left(\frac{T}{m}\right)^{k},
$$

where $\eta_{i}$ are bulk points near the singularity lying on the bouncing geodesic and $R^{(k)}$ is shorthand for a curvature invariant that has scaling dimension (length) ${ }^{-2 k}$. Because the only length scale near the singularity is $l$, the shortest proper time between the geodesic and the singularity, we have $R^{(k)} \sim l^{-2 k}$. The region of proper distance $T$ where the geodesic is near the singularity is also $\sim l$. Putting in these estimates, (4.2) becomes

$$
\left\langle\phi\left(\eta_{1}\right) \phi\left(\eta_{2}\right)\right\rangle \sim \sum_{k=0}^{\infty} c_{k}\left(\frac{1}{m l}\right)^{k} .
$$


This is reasonable since $m l$ is the only dimensionless combination available near the singularity. From (2.16) we see that $r_{\min } \sim 1 / E$ and $E \sim 1 /\left(t-t_{c}\right)$. It follows immediately from the metric that $l \sim r_{\text {min }}^{2} \sim\left(t-t_{c}\right)^{2}$. So we expect the $1 / m$ expansion to have the structure

$$
\langle\phi \phi\rangle(t) \sim \sum_{k=0}^{\infty} c_{k}\left(\frac{1}{m\left(t-t_{c}\right)^{2}}\right)^{k} .
$$

We should be able to compute these coefficient functions on the primary sheet and the analytically continue them to the secondary sheet to study their singular behavior. This gives an example of recovering nontrivial information about the neighborhood of the singularity from outside the horizon correlators. The information here is just the diverging curvature near the singularity, and it is clear from the above that the main contribution to the singular behavior comes from distances $\sim l$ which can be made arbitrarily short (in supergravity approximation) by taking $t \rightarrow t_{c}$. It is possible that the strengthening of the singularity with increasing $k$ will have a recognizable signature in the correlator on the first sheet.

\subsection{Finite $\alpha^{\prime}$}

We now go beyond the supergravity approximation by letting the string length $l_{s}$, $\left(\alpha^{\prime} \sim l_{s}^{2}\right)$ be finite. In the boundary SYM theory, this corresponds to taking the 't Hooft coupling $\lambda=g_{\mathrm{YM}}^{2} N=\left(R / l_{s}\right)^{4}$ finite. We still work in the classical limit $g_{s} \rightarrow 0, N \rightarrow \infty$.

When $l_{s}$ is finite, the behavior of correlators in the large $m$ limit depends on which large $m$ excitation we are studying. If we take a generic perturbative string state with many oscillator modes excited then the size of the excitation will diverge as $m \rightarrow \infty$ and the correlator will cease to serve as a local probe. Instead we consider D-branes, which, at $g_{s}=0$ are essentially pointlike, infinitely massive objects [51,52,53. In particular in the $A d S_{5} \times S^{5}$ IIB string theory we study D3-branes wrapped around an $S^{3}$ submanifold of $S^{5}$. Such a state is pointlike in the $A d S_{5}$ space and realizes the BPS state carrying $\mathrm{SO}(6)$ charge, for large charge. These are the giant gravitons 47.

The main effect of small but finite $l_{s}$ on such states is encapsulated in corrections to the supergravity Lagrangian. These include, for instance, corrections of the form $\left(\alpha^{\prime}\right)^{k} R^{(k)}$. The small parameter controlling the size of such terms is $l_{s} / l$. For phenomena near the horizon $l \sim R$ and this small parameter is of order $l_{s} / R \sim 1 / \lambda^{1 / 4}$. Such terms have a number of effects on the dynamics, including small shifts of the metric and dilaton fields [54].

We can estimate the effect of such shifts on the geodesic dynamics by following the discussion in the previous section about the finite mass black hole. Just as in that situation, the most general small, smooth shift in the geodesic equations can be encapsulated by 
deforming (3.11) and (2.18) into

$$
\begin{aligned}
t & =E^{3}-a E \\
\mathcal{L} & =-E^{4}-b E^{2}
\end{aligned}
$$

Here $a, b$ are small parameters whose signs are undetermined. As in the discussion following (3.13) and (3.12) this structure guarantees that the bifurcation, needed for analytic continuation, does not disappear at small but finite $l_{s}$. At most the cube root singularity at $t=0$ splits into two square root singularities.

This persistence of the singularity at small $t$ creates a puzzle. Explicit field theory calculation of the two point correlator at small $\lambda$ (i.e., large $l_{s}$ ) shows no sign of such a singularity. So it seems there must be an $m=\infty$ phase transition at finite $\lambda$. Perhaps we have missed some effect at large $\lambda$ that removes the singularity. On the other hand it does not look difficult to construct metric and dilaton modifications that remove the singularity for a finite size deformation.

The nature of $l_{s}$ corrections for $t$ near $t_{c}$ gives information about stringy behavior near the black hole singularity. As discussed above, we expect corrections to be controlled by the dimensionless ratio $l_{s} / l=\lambda^{1 / 4} /\left(t-t_{c}\right)^{2}$. So there should be large corrections for $t \sim t_{c}$.

Of course, this is a string tree level effect and can in principle be computed by string world sheet techniques. The D-brane trajectory is encoded in a boundary state. The analytic continuation necessary to study the real geodesic requires boundary states with complex D-brane positions. Such states have been studied recently in other contexts [55] [56]. Perhaps one can circumvent the analytic continuation by directly formulating the conformal field theory in Lorentzian space, and then selecting the boundary state corresponding to a D-brane following the real geodesic.

\subsection{Finite $g_{s}$}

We now relax the last remaining constraint and consider finite $g_{s}$. A problem immediately arises because the masses of D-branes are $\sim 1 / g_{s}$ and so the mass $m$ of the probe particle cannot be taken infinitely large. More precisely if the $S^{3}$ radius on which the $D 3$-brane is wrapped is $\sim R$, then its mass $m$ is $m R \sim\left(m_{s} R\right)^{4} / g_{s} \sim \lambda / g_{s} \sim N$. If the $S^{3}$ radius is string scale then $m R \sim m_{s} R / g_{s} \sim N^{1 / 4}$. If the $\operatorname{SO}(6)$ charge is $Q$ then $m R=Q .9$ The stringy exclusion principle [57] and the giant graviton analysis reviewed above show that stability requires $Q \leq N$. As discussed above, at finite $m$ the correlation function

9 This is only approximately true because supersymmetry is broken in the black hole background, and the BPS conditions no longer strictly apply. But the semiclassical analysis in [47] demonstrates that this relation continues to be approximately true for large $Q$. 
contribution from the dominant saddle is "contaminated" by $e^{-m}$ corrections from subdominant saddles that will eventually become dominant on crossing an anti-Stokes line. We can certainly study correlators as a power series in $g_{s}$ since $g_{s}^{k} \gg e^{-m} \sim e^{-\lambda / g_{s}}$ as $g_{s} \rightarrow 0$. Because the emission and absorption of virtual perturbative quanta cannot significantly alter the D-brane trajectory, we should be able to analytically continue the geodesic through the anti-Stokes line. This will allow us, in principle, to study the nature of string perturbation theory in the vicinity of the singularity by continuing each order across the anti-Stokes lines to $t \rightarrow t_{c}$. Again, these quantities should in principle be computable by studying higher genus string diagrams with the appropriate boundary state. Of course there will be $1 / m$ corrections to these order $g_{s}^{k}$ amplitudes which can be numerically larger than higher order $g_{s}$ corrections. But these are fluctuations about the main saddle, and will each analytically continue to the desired real geodesic. They can be distinguished from $g_{s}$ effects by their different parametric dependence on $\lambda$, or equivalently $Q$.

In the supergravity approximation at least, we might expect divergences in loop amplitudes when interaction vertices approach the singularity. This issue was discussed for $d=3$ in [31]. On general grounds, applicable here as well, such divergences should not occur. The correlators are manifestly finite in Euclidean SYM. They are analytic and so can be singular at most on a set of complex codimension one. So a generic divergence is not possible. From the bulk point of view the potential divergences in $d=3$ are not present because the analytic continuation from Euclidean space induces a shift $r \rightarrow r+i \epsilon$ which regulates the singularity. Interaction points are integrated across the singularity into another region to implement this. Remaining imaginary parts are cancelled between past and future singularities. Although $d>3$ is more complicated we think it reasonable to expect a similar mechanism here.

But the elimination of divergences need not eliminate large finite contributions. Since the geodesic near $t_{c}$ is very close to one singularity it is natural to expect large finite contributions in quantum corrections. We think this is what is being computed by the boundary theory near $t_{c}$.

We would like, at least in principle, to be able to obtain nonperturbative information about the singularity from the nonperturbatively well defined boundary CFT.

Operationally, the size of nonperturbative phenomena whose continuation we want to study must be parametrically larger at the point we calculate than the $e^{-m}$ contamination from the subdominant saddles that would become dominant at an anti-Stokes line and prevent analytic continuation of the "metastable" phase. For example, suppose we wish to study D-brane effects, for instance the pair production of small D-branes. Such effects are typically of size $e^{-1 / g_{s}}$. Using the largest stable giant graviton as a probe, $e^{-m} \sim e^{-\lambda / g_{s}}$. Since $e^{-1 / g_{s}}$ is parametrically larger than $e^{-\lambda / g_{s}}$, it should be possible to reliably compute such processes and then analytically continue them to the real sheet. Processes that may 
be impossible to compute include NS brane production, expected to be of order $e^{-1 / g_{s}^{2}}$. It may be possible, though, to isolate these effects by studying the boundary theory as an analytic function of $g_{s}$ and $\lambda$.

Processes where the nonperturbative effects are dominant should be much easier to study. As a first example, consider D-instanton effects that are the leading contribution to certain anomalous processes involving the bulk axion, related to the boundary $\theta$ parameter. The effect of $\theta$ on giant graviton correlators should behave like $e^{-1 / g_{s}}\left(f_{0}(t)+O\left(e^{-m}\right)\right)$. Here we have normalized to the $\theta=0$ answer. The function $f_{0}(t)$ can in principle be computed on the primary sheet with parametrically small error. Then it can be continued to the secondary, real sheet. If D-instanton effects are enhanced near the singularity then there should be a signal in $f_{0}(t)$ near $t_{c}$.

As a second example, consider a charge $Q$ wrapped D-brane "particle" of mass $m R=$ $Q$. Take $Q \sim N$. Now imagine a process where this particle fragments into two other such particles of charges $Q_{1}$ and $Q_{2}$, with $Q_{1}+Q_{2}=Q$ and $Q_{i} \sim Q / 2$. The black hole is not a supersymmetric background so there is no BPS condition that prevents this. But this process is certainly nonperturbative. By considering the amount of D-brane that must be created and destroyed we have provisionally estimated that the rate for this process is $\sim \exp (-Q) 10$ Both initial and final particles follow geodesics of the kind we have been discussing, assuming that the final boundary operators at placed at the same point. These geodesics should continue smoothly onto the secondary sheet. Schematically, we expect the amplitude for this process to look like $e^{-Q}\left(f_{0}(t)+O\left(e^{-Q}\right)\right)$ where we have normalized to the two particle charge $Q$ correlator. Again, $f_{0}(t)$ is in principle calculable on the primary sheet with parametrically small error. It can then be analytically continued to $t_{c}$.

The kind of quantum information that can be obtained results from taking $g_{s} \rightarrow 0$ in a certain way. If one focuses on the behavior of amplitudes as $t \rightarrow t_{c}$ then perhaps the information that can be extracted about the singularity is some kind of double scaling limit that compensates for a shrinking $g_{s}$ by examining processes that grow large for $t \rightarrow t_{c}$.

\section{Discussion}

The analysis of the preceding sections shows that a significant amount of information from behind the horizon, and in particular from the region near the singularity, is encoded

10 In this entire discussion we have not considered the D-brane wrapped on a sphere in $\mathrm{AdS}_{5}$ discussed [58,59]. Such a state gets light near the black hole singularity and so it should have a strong effect on the analytically continued dynamics. It may be that the nonperturbative tunneling to this configuration discussed in [58,59] is the dominant mechanism for fragmentation near the black hole singularity. This deserves more study. 
into boundary CFT correlators. An initial question we must consider is how this information can emerge from the hot, thermal, apparently featureless horizon. Our understanding of this issue is limited, but it is clear that analyticity plays a central role. It is analyticity that lets us reliably follow a geodesic into a region where its contribution is exponentially subdominant. One might think that our results are merely a consequence of the analytic nature of the classical AdS Schwarzschild geometry, which allows one to compute the complete metric from a small sample of it far outside the horizon. But clearly more than this is involved. We have argued in the previous section that a large amount of stringy and quantum information should be accessible by analytic continuation as well. It seems that it is the analyticity of the full quantum theory, or at least a limiting part of it, that is the central issue.

Analyticity is not a central notion in classical general relativity. For instance, in the collapse of a matter shell to form a black hole the metric is not analytic, and only one asymptotic region exists. But outside the shell the metric is just the standard black hole metric. Correlators will in general not be analytic in such a spacetime. It seems that our methods may not apply to such cases. Even with analytic initial data, the same side correlators in a collapse scenario seem to differ from those of the eternal black hole by exponentially small terms, even though the geometry behind the horizon differs markedly.

However, analyticity does play a central role in quantum field theory. Multipoint correlation functions are analytic functions of the point locations. So quantities computed in AdS/CFT will generically be analytic. We can envision setting up a collapse scenario in AdS/CFT by applying a large number of boundary operators at a fixed time far in the past. We then let this matter evolve. From the boundary point of view, we excite many gluons and then let them thermalize. We have created one of the microstates counted in the black hole entropy. We then can insert two very massive particle boundary operators as probes. Their correlator will be analytic in their time separation, and should go over to the thermal ensemble in the limit of large energy, which corresponds to large black hole mass. But even away from this limit the probe correlator is precisely analytic. In principle, we can test for periodicity in imaginary time, remnants of the $t=0$ branch point and of the $t_{c}$ singularity. We can look for exponentially small one sided contributions that become significant when continued to opposite sides. It may well be difficult to disentangle these small terms from the $e^{-m}$ contamination that obstructs analytic continuation. The different parametric dependence and ability to study different microstates might make it possible to distinguish the two. This question may be easier to study in $d=3$ where the real behind the horizon geodesic dominates for all $t$. If the effective geometry behind the horizon is modified then it should make a large effect on this two sided correlator.

These questions should be related to the issue of recovering information that has fallen into the black hole. The lack of information loss in eternal black holes is signalled 


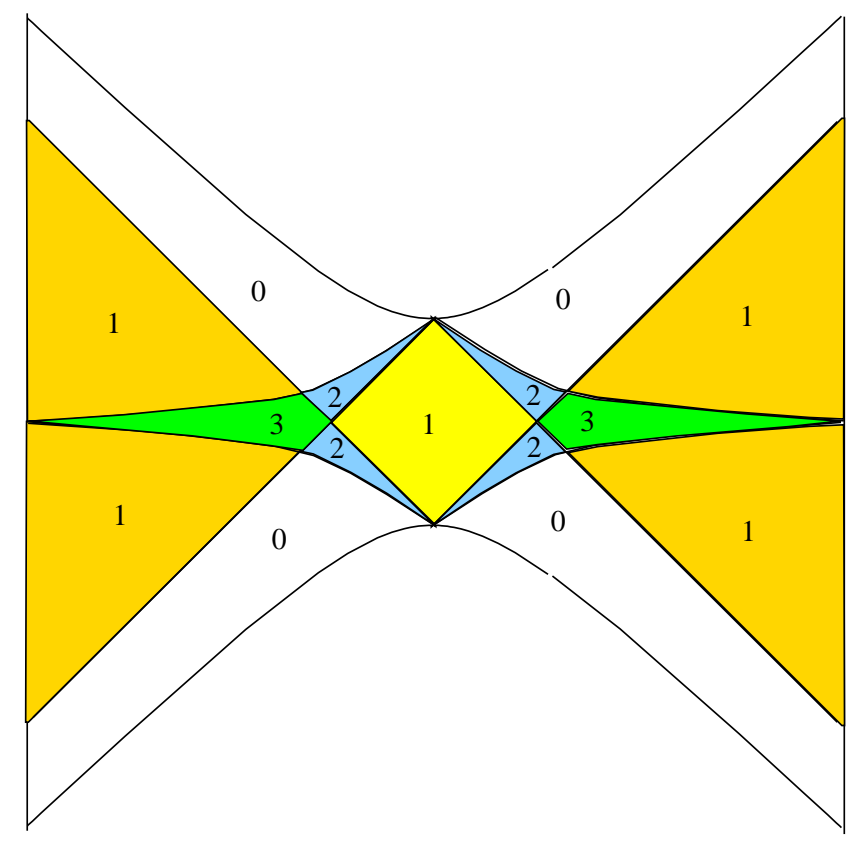

Fig. 12: Symmetric spacelike geodesics of AdS Schwarzschild. The numbers indicate how many such geodesics pass through each point in that region.

by Poincaré recurrences [30], which occur on time scales $t_{r}$ exponentially longer than $t_{c}$, $t_{r} \sim \exp \left(N^{2}\right) t_{c}$. These recurrences may correspond to large fluctuations in the classical geometry [30]. These occur at times $\sim N^{2} t_{c}$. It would be very interesting to connect these ideas to those discussed in this paper, although the enormous difference in time scales makes this challenging.

As mentioned in Section 3, tracking the bouncing geodesic through the anti-Stokes line is a bit like following a metastable phase in a statistical mechanical system, although this analogy is imprecise. A related phenomenon has already been discussed in the AdS/CFT correspondence. Gross and Ooguri [60] considered the expectation value of two facing Wilson loops of radius $R$ and separation $L$. In the limit of infinite string tension $\lambda \rightarrow \infty$ this quantity is determined by the minimal area string world sheet spanning the loops. As $L$ is increased this surface jumps in a discontinuous, "first order" transition. At large $L$ the disconnected surface dominates. But one can in principle continue through this "antiStokes line" and study the metastable connected surface until it pinches off into a kind of singularity. It would be interesting to consider various kinds of fluctuation corrections in this situation and see which ones can be continued through the anti-Stokes line to the pinch off point.

We have argued that the real geodesic (which becomes almost null at $t=t_{c}$ ), contributes to boundary correlators in a subtle subleading way that can be exposed by analytic continuation to a secondary sheet. We might ask whether it ever provides the dominant 
contribution to a physical quantity. We are not sure of the answer to this question. But, if we temporarily put aside the well founded concerns about measuring local bulk correlation functions in quantum gravity, and just compute them in classical supergravity approximation, we can find some answers. In Fig.12 we have displayed the regions for which a symmetric bulk two point function is dominated by various geodesics. Inside regions 3,2 , and the central 1 , the most likely scenario is that the geodesic that dominates the correlation function lies on the real branch (real $E$ ) and so will evolve into the nearly null geodesic. For the dominant geodesic to get within a proper time $l$ of the singularity, the correlator points have to be separated by a proper distance $\sim l$. For these quantities the large stringy and quantum effects discussed in the previous section should be important factors in computing the leading result, not a subtle subleading contribution. But of course the role of such bulk quantities is a deeply confusing one.

We now ask what this new information can tell us about the singularity. Unfortunately, little is known about boundary CFT correlators at strong coupling. In fact, our results in the bulk make many new predictions about them, as has often been the case in AdS/CFT. Still, it is a useful exercise to ask what could be learned if complete correlator information were available for the CFT.

In previous sections we concentrated on the two point correlator. But there is no reason not to study more general correlators (an example of this was discussed in Section 4). In particular, consider the correlator with $K$ operators on the asymptotic boundary of region I and $K^{\prime}$ on the boundary of region III, with a variety of $S O(6)$ charge assignments. Each operator corresponds to a large mass particle (wrapped D-brane), and can be inserted at different points on the AdS sphere. Schwarzschild time runs in opposite directions in the two asymptotic regions, so the observer in region I can prepare an in-state of $\mathrm{K}$ particles, send the beam through the horizon, and then the other observer can observe an outstate of $K^{\prime}$ particles, assuming that we have analytically continued so the bounce geodesic dominates. If the boundary times of these operators are all near $t_{c}$, the geodesics are almost null and hence almost on shell. A "meta-observer" could in principle compare the data of these two observers and compute a "meta S-matrix." This quantity would seem to contain significant information about the fate of organized matter as it approaches the singularity.

But from the point of view of a global observer using Kruskal time, these correlators correspond to particle-antiparticle annihilation near the singularity, and the correlator is a kind of vacuum persistence amplitude. Its interpretation is less clear.

A question one typically asks about singularities in consistent physical theories is what resolves them. Here the black hole singularity seems deeply connected to the boundary $t_{c}$ singularity in the analytically continued CFT amplitudes. Perhaps this $t_{c}$ singularity is smoothed out in the full CFT with finite $\lambda$ and $g_{s}$. Of course the difficulties described in 
the previous section in extracting finite $g_{s}$ information make it possible that this question can only be addressed in some double scaling limit.

The definition of gauge theory correlators by analytic continuation from the Euclidean thermal theory must determine some boundary conditions for bulk fields at the black hole singularity. Some work has been done on this question in the $d=3$ case [61, but $d>3$ is technically more difficult. We should be able to address this issue by tying geodesic techniques to WKB approximations of the wave equation. This may be connected to the possibility of defining different black hole systems by starting with different initial states than the Hartle-Hawking state.

One important tool for analyzing the boundary CFT is to go to small 't Hooft coupling $\lambda($ at $N=\infty$ ) where weak coupling perturbation theory is valid. But, as mentioned in Section 4.2, at weak coupling there is no sign of the branch cut signalling the metastable phase on which we concentrate. As noted above it is not difficult to imagine $l_{s}$ corrections to the metric and other supergravity fields that will remove the branch point and bouncing geodesic. It would be very useful to have an understanding of this from the gauge theory side.

\section{Acknowledgements}

We would like to thank Ben Craps, Ben Freivogel, Gary Horowitz, Jared Kaplan, Per Kraus, David Kutasov, Emil Martinec, Rob Myers, Mukund Rangamani, Simon Ross, Andrei Starinets, and Lenny Susskind for helpful discussions. This work is supported in part by NSF grant PHY-9870115 and by the Stanford Institute for Theoretical Physics. Matthew Kleban is the Mellam Family Foundation Graduate Fellow.

\section{Appendix A. Kruskal coordinates}

We have motivated the non-square nature of the Penrose diagram by studying radial null geodesics. Since the Schwarzschild coordinates do not cover the whole spacetime, we have used complexified coordinates in order to consider the globally extended spacetime. However, for the purposes of understanding the global structure, it is more desirable to pass to real, globally-defined coordinates. The most convenient ones to use are the Kruskal coordinates, which we now present. This will allow us to see the behavior of null geodesics, without having to use complexified coordinates and to deal with coordinate singularities at the horizons.

Many of the issues discussed in this and the next Appendix have previously been addressed in a general context in [46] . 
Suppressing the angular directions, the metric (2.2) is

$$
d s^{2}=-f(r) d t^{2}+\frac{d r^{2}}{f(r)}
$$

with $f=r^{2}-\frac{1}{r^{2}}$. Now define the Tortoise coordinate

$$
r_{*}=\int_{0}^{r} \frac{d r^{\prime}}{f\left(r^{\prime}\right)}+C
$$

with $\mathrm{C}$ an integration constant we will choose later, so as to make the Kruskal coordinates everywhere real. Letting $u=t-r_{*}, v=t+r_{*}$, we have $d s^{2}=-f d u d v$. This is singular at the horizon since the determinant vanishes; but we can perform a further coordinate transformation $U=-e^{-2 u}, V=e^{2 v}$ which will cast the metric into an extendible form. In these new coordinates, with $f(r)$ and $r_{*}(r)$ being implicitly functions of $U$ and $V$, we have

$$
d s^{2}=-\frac{f}{4} e^{-4 r_{*}} d U d V
$$

Letting $U=T-X$ and $V=T+X$, we can write this in a more familiar form $d s^{2}=$ $g(T, X)\left(-d T^{2}+d X^{2}\right)$. Note that by our choice of coordinates $U(u)$ and $V(v)$, the factor $g(T, X)$ in front of $\left(-d T^{2}+d X^{2}\right)$ is nonsingular at the horizon: although $f$ by itself vanishes at the horizon, the $e^{-4 r_{*}}$ factor cancels this, so that the conformal factor $g$ remains finite across the horizon. Therefore, we can extend $(U, V)$ from $U<0, V>0$, to $U, V \in$ $(-\infty, \infty)$, subject to the constraint $0<r<\infty$.

We easily derive the transformation laws 1 l between the old and the new coordinates:

$$
\begin{gathered}
-e^{4 r_{*}(r)}=T^{2}-X^{2} \\
2 t=\tanh ^{-1} \frac{T}{X}
\end{gathered}
$$

Note that $r_{*}$ acquires an imaginary part as the integral passes through the pole at 1 . It is correspondingly convenient to define $C=\frac{i \pi}{4}$, which makes $r_{*}$ real outside the horizon. More specifically, we have

$$
e^{4 r_{*}}=\left(\frac{r-1}{r+1}\right) e^{2 \tan ^{-1} r}
$$

Using (A.4), we can now read off the curves of constant $X^{2}-T^{2}$ labeling a constant $r$ surface. In particular, it is easy to see that the singularity $(r=0)$ corresponds to $T^{2}-X^{2}=1$, while the boundary $(r \rightarrow \infty)$ of AdS Schwarzschild is given by $X^{2}-T^{2}=e^{\pi}$.

11 For general functions $f(r)$ in (A.1) with simple zeros at $r=r_{+}$, we would have $T^{2}-X^{2}=$ $-e^{f^{\prime}\left(r_{+}\right) r_{*}(r)}$ and $2 \tanh ^{-1} \frac{T}{X}=f^{\prime}\left(r_{+}\right) t$. 


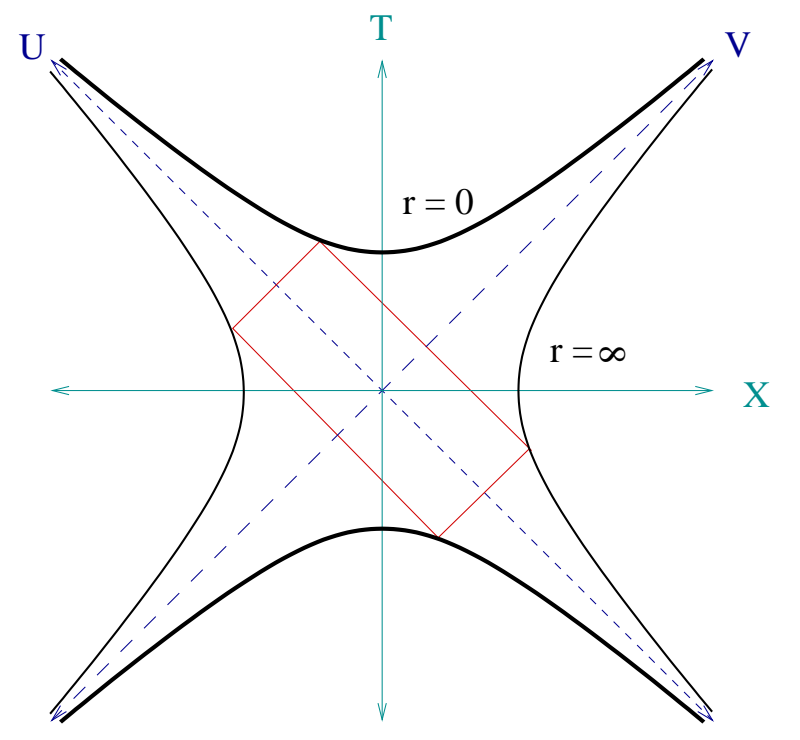

(a)

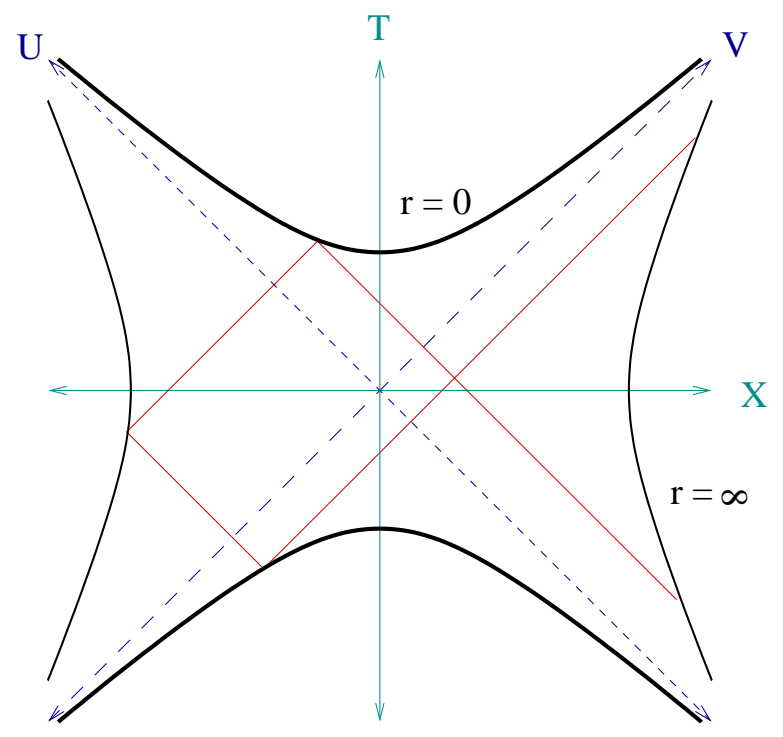

(b)

Fig. 13: Kruskal diagrams for AdS Schwarzschild, with a) $d=3$ and b) $d>3$. Radial null curves lie at 45 degrees; the horizons are represented by the diagonal dashed lines. The thick (spacelike) hyperbolas correspond to the singularity, the timelike ones to the boundary. The crucial difference between three and higher dimensions is that in the former case, the hyperbolas are the same distance from the origin, which translates into the geometric property that any radial null curve bouncing around the diagram ends up at the same point it started at. In higher dimensions this doesn't happen.

Note that each of these equations is satisfied by two disjoint curves, related to each other by $X \rightarrow-X, T \rightarrow-T$. The full spacetime is then bounded by the four hyperbolas in Kruskal coordinates,

$$
-e^{\pi}<T^{2}-X^{2}=U V<1
$$

where the lower bound corresponds to the asymptotic (timelike) boundary, and the upper bound to the (spacelike) singularity. This is to be contrasted with the corresponding situation for the $d=3$ BTZ spacetime, where a similar analysis would yield $-1<T^{2}-$ $X^{2}=U V<1$.

Fig.13 illustrates the contrast between the Kruskal diagram in $d=3$ and $d>3$ dimensions. For the 3 dimensional BTZ spacetime, sketched in Fig.13a, the singularities and boundaries intersect the Kruskal coordinate axes at the same distance. On the other hand, in all higher dimensions, the Kruskal diagram is as shown in Fig.13b, where the singularities come closer to the center of the diagram than the boundaries. As we just computed, for the $d=5$ large black hole, the ratio of these distances is $e^{\pi}>1$. 
This immediately implies that there cannot be a radial null geodesic starting on the boundary at $t=0$ (which implies $T=0$ ) and hitting the singularity in the middle $(X=0)$. A more invariant way to say this is by considering a radial null geodesic (or a sequence of geodesics), which "bounce around 12 the diagram", as shown by the thin solid diagonal lines in Fig.13. It is easy to see that if the hyperbolas intersect at the same distance, as in Fig.13a, then such a sequence of null curves ends up at exactly the same point where it started; otherwise, if the hypebolas intersect at different distances as in Fig.13b, the geodesics end at a different point. Furthermore, if the boundaries are further than the singularities, these curves intersect inside the spacetime. As we will discuss in Appendix $\mathrm{B}$, this simple geometrical property proves, in a more invariant way, that the Penrose diagram of AdS Schwarzschild cannot be drawn as a square for $d>3$, but can for $d=3$.

So far, we have presented our results only in the large black hole limit. One may wonder whether for black holes comparable to (or much smaller than) the AdS radius, this effect does not go away. In fact, as we now explain, it gets larger. The smallest value that the magnitude of the lower bound in (A.6) can have occurs in the large mass limit, where this goes to $X^{2}-T^{2}=e^{\pi}$. For smaller black holes this value increases, and in fact gets arbitrarily large in the small mass limit: the boundary is then given by $X^{2}-T^{2}=e^{\pi \frac{R}{r_{+}}}$ with $r_{+}$and $R$ denoting the black hole and AdS radius, respectively. As we discuss next, on a Penrose diagram, such as sketched in Fig.2b, the smaller black holes would have the singularities more bowed in (using the same conformal rescaling), or equivalently the boundaries more bowed out in Fig.2c. But this is exactly what we would expect if we naively cut-off the asymptotically flat Schwarzschild Penrose diagram at larger and larger distances $R$ (effectively corresponding to smaller and smaller black holes).

\section{Appendix B. Penrose diagram}

We have already discussed the qualitative features of the causal structure of AdS Schwarzschild black holes, but let us now confirm this by finding the explicit Penrose diagrams for these spacetimes. This will be achieved by finding appropriate transformation $V \rightarrow \tilde{v}(V)$ and $U \rightarrow \tilde{u}(U)$ of the Kruskal coordinates $(V, U)$ (this guarantees that radial null geodesics will be 45 degree lines), such that the boundaries of the spacetime now lie at finite coordinate distance.

12 Specifically, starting from any point on the right boundary, consider a future-directed ingoing null geodesic; from where this hits the future singularity, draw the past-directed outgoing null curve which eventually hits the left boundary; then the past-directed ingoing null curve which hits the past singularity; and finally the future-directed outgoing null curve which comes back to the right boundary again. 


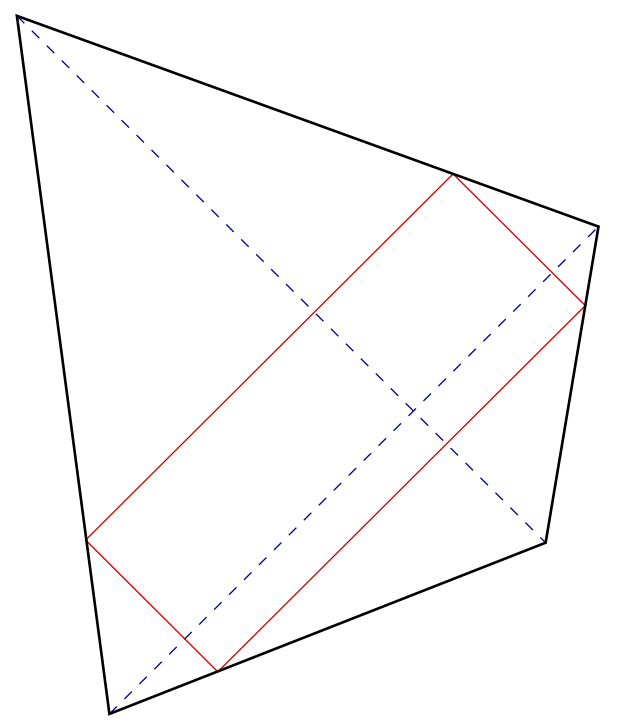

Fig. 14: Most general form of Penrose diagram with timelike boundaries and spacelike singularities being straight (thick lines on the diagram), since the horizons (dashed 45 degree lines) must join the opposite vertices. A null ray bouncing around (solid thin line) must come back to the same point.

For example, letting $V=\tan \frac{\tilde{v}}{2}$ and $U=\tan \frac{\tilde{u}}{2}$ compactifies the spacetime into a region inside $\tilde{v}, \tilde{u} \in(-\pi, \pi)$. Letting $\tilde{v}=\tau+\rho$ and $\tilde{u}=\tau-\rho$, we can now check that the singularities $U V=1$ are the straight lines $\tau= \pm \frac{\pi}{2}, \rho \in\left(-\frac{\pi}{2}, \frac{\pi}{2}\right)$. However, the boundaries, given by $\left(\tan \frac{\tau+\rho}{2} \tan \frac{\tau-\rho}{2}\right)=-e^{\pi}$, in the allowed region $\tilde{v}, \tilde{u} \in(-\pi, \pi)$, can be explicitly checked to be "bowed out". On the other hand, if we try to straighten out the boundary, e.g. by taking $V=e^{\frac{\pi}{2}} \tan \frac{\tilde{v}}{2}$ and $U=e^{\frac{\pi}{2}} \tan \frac{\tilde{u}}{2}$, so that $U V=-e^{\pi}$ is given by $\rho= \pm \frac{\pi}{2}, \tau \in\left(-\frac{\pi}{2}, \frac{\pi}{2}\right)$, we find that the singularity must be "bowed in". An exact plot (generated by the latter transformation) was presented in Fig.5b.

One may ask whether one can make a more clever choice of $\tilde{u}$ and $\tilde{v}$ so as to straighten out both the singularity and the boundary. After all, the usual lore that "curves can be straightened out by suitable conformal transformations" should apply here as well. But in fact, we see that the present problem is sufficiently constrained so that this cannot happen.

We can make a general geometric argument for why the singularities and the boundaries cannot be drawn as straight lines, with the whole spacetime filling some compact region on the diagram. The first ingredient is to note that the horizons must be 45 degree lines, and that the singularities and boundaries must "meet" at these. 3 (Note that

13 By definition, horizons are the boundaries of the past of infinity (so the boundary must touch the horizon on the Penrose diagram), and any causal curve which enters the black hole must end at the singularity (so similarly, the singularity must touch the horizon in a Penrose diagram); and finally, the horizons themselves cannot form part of the boundary of the spacetime, as by 
this is the reason the Penrose diagram could not be e.g. a rectangle, which might naively seem consistent with the observed properties of null geodesics.) The most general way the corresponding Penrose diagram with straight singularities and boundaries could look is sketched in Fig.14. But one can easily show by a series of similar-triangle arguments that a null curve bouncing around this diagram (analogously to that sketched in Fig.13) must come back to the same point. This is in contradiction to the properties of null geodesics, as we have demonstrated above, so the singularities and boundaries cannot both be straight, if the spacetime is to be mapped into a compact region.

The last requirement is very important: we can easily construct maps which make both singularities and the boundaries straight, but at the cost of the "center" (where the two horizons intersect) being mapped to infinity. One such example is to let $U=\tilde{u}$ and $V=1 / \tilde{v}$. The diagram of the spacetime would then not be compact, and would not qualify as a Penrose diagram. In retrospect, it is clear that one can't straighten out any two curves by conformal transformation, without letting them go to infinity. For example, consider two distinct curves which have the same endpoints. Whatever conformal transformation we apply, the two curves will still have the same endpoints. But clearly, in the flat geometry there is only a single straight line between any two points, so if the conformal transformation straightens out both curves without making them go off to infinity, it must be singular (i.e. non-invertible).

We should make one cautionary remark: we have shown that, keeping the boundaries straight, the singularities must be bowed in. The amount of bowing, however, does depend on the conformal transformation used. In other words, the crucial geometrical property we used was that radial null geodesics bouncing around the diagram end up at a different point from which they started. However, by a suitable conformal transformation, we can bring this point to appear arbitrarily close to the starting point, effectively making the Penrose diagram look almost, but not quite, like a square.

\section{Appendix C. Spacelike geodesics with angular momentum}

In this paper, we have been using only radial geodesics in AdS Schwarzschild. For completeness, let us now consider geodesics to ones carrying some "angular" momentum.14 In this case, the behaviour of spacelike geodesics is altered significantly. In addition to timelike and radial components, the geodesics have an angular component, described by

definition they lie entirely in the interior.

14 We will use notation appropriate to finite-sized (spherically symmetric) AdS Schwarzschild black hole; in the large black hole limit, the angular momentum becomes the linear momentum along one of the translationally invariant directions. 
the constant of motion $L=\dot{\phi} r^{2}$. The radial equation (2.10) is now modified to

$$
\dot{r}^{2}=E^{2}+f(r)\left(1-\frac{L^{2}}{r^{2}}\right)
$$

which describes a 1-dimensional motion of a particle of energy $E^{2}$ in the effective potential

$$
V_{\mathrm{eff}}(r)=-r^{2}+L^{2}+\frac{1}{r^{2}}-\frac{L^{2}}{r^{4}}
$$

We see that rather than being repelled by the spacelike singularity, the spacelike geodesics which penetrate sufficiently close to the singularity are now attracted, and therefore terminate at the singularity. Namely, a spacelike geodesic with energy $E$ and angular momentum $L$ starting at $r=\infty$ can propagate to $r=r_{i}$, where $r_{i}$ is now a function of both $E$ and $L$, given by the solution of the equation

$$
E^{2}+f\left(r_{i}\right)\left(1-\frac{L^{2}}{r_{i}^{2}}\right)=0
$$

Note that for $E=0$, for any $L, r_{i}=1$ as before (assuming $L<1$ ); however for $E>0$ the behaviour is more complicated, and depends on $L$. For $L>1$, we see that $r_{i}>1$ since otherwise the LHS of (C.3) would be strictly positive. This means that such geodesics can never cross the horizon, and in particular can never connect the two boundaries of AdS Schwarzschild. Thus they are not relevant for us, and we will henceforth consider only geodesics with $L \leq 1$. For $L<1$, the geodesics must propagate past the horizon (otherwise the LHS of (C.3) would again remain positive), and there are now two possibilities: for high enough energy, the geodesic terminates at the singularity, whereas for energies below this critical energy, $E<E_{c}$, it reaches only down to $r_{i}>L>0$, and reemerges in the other asymptotic region. The dividing energy $E_{c}$ is of course given by the maximum value of the effective potential in (C.2), $E_{c}^{2}=f\left(r_{i}\right)\left(\frac{L^{2}}{r_{i}^{2}}-1\right)$. Note that this is consistent with our previous picture, since $E_{c} \rightarrow \infty$ as $r_{i} \rightarrow 0$, i.e. as $L \rightarrow 0^{+}$. In the small $L$ limit, this behaves as $E_{c} \sim \frac{1}{L R}$.

Confining attention to $L \leq 1$ and $E \leq E_{c}$, let us consider the time $t_{0}$ at which a spacelike geodesic of given $E$ and $L$ has to start in order to be symmetric. By the same arguments as before, this is given by

$$
t_{0}(E, L)+i \beta / 4=-\int_{r_{i}(E, L)}^{\infty} \frac{E}{f(r) \sqrt{E^{2}+f(r)\left(1-\frac{L^{2}}{r^{2}}\right)}} d r
$$

As before, $t_{0}$ vanishes for $E=0$, and becomes more negative as $E$ increases. Thus, to see the possible range of $t_{0}$ for various allowed values of $E$ and $L$, it suffices to consider 
the part of the integral around $r_{i}$ for energies close to the saturating value $E_{c}$. Since the effective potential peaks at $E_{c}$, and $r_{i}\left(E \rightarrow E_{c}\right)$ approaches the $r$-value at which $V_{\text {eff }}$ attains its maximum, we can approximate the effective potential near its maximum by $V_{\text {eff }}=-f(r)\left(1-\frac{L^{2}}{r^{2}}\right) \approx E_{c}^{2}-\mu^{2}\left(r-r_{i}\right)^{2}$, where the coefficient $\mu^{2}$ is some complicated function of $L$ and the spacetime parameters. Substituting this back into (C.4), we see that for energies $E \approx E_{c}$, the integrand becomes $\frac{E_{c} / f(r)}{\left(r-r_{i}\right)}$. Since $f(r)$ is well-behaved at $r=r_{i}$ for finite $L$, we see that the integral (C.4) has logarithmic divergence at $r_{i}$. Thus, unlike in the $L=0$ case where $t_{0}$ was bounded by $t_{c}(\mathrm{cf}$. (2.16)), for $L>0$ this bound disappears, and $t_{0}$ can have arbitrary values.

Let us now consider the behavior of the proper length along these symmetric spacelike geodesics carrying angular momentum. This is given by the generalization of (2.17), namely

$$
\mathcal{L}=2 \int_{r_{i}}^{r_{\max }} \frac{d r}{\sqrt{E^{2}+f(r)\left(1-\frac{L^{2}}{r^{2}}\right)}}
$$

where $r_{\max }$ is the upper cutoff and $r_{i}$ is given by solution of (C.3). Since we already analyzed the behavior as $E$ increases, for simplicity we will now consider the $E=0$ case, to see how increasing $L$ affects the proper length $\mathcal{L}$. For $E=0, r_{i}=1$ independently of $L$, so we clearly see that increasing $L$ must increase the proper length $\mathcal{L}$ (since the denominator of the integrand in (C.5) decreases). In fact, as $L \rightarrow 1$, it is easy to see that the length diverges logarithmically at the near-horizon region.

\section{Appendix D. Derivation of the geodesic time $t(E)$}

In this appendix we derive the formula for the boundary time $t_{0}$ as a function of $E$ for the case of the symmetric spacelike geodesics. We first look at the infinitely massive black hole. The integral to be done is 2.15,

$$
-t_{0}=\int_{r_{i}}^{\infty} \frac{E}{f(r) \sqrt{E^{2}+f(r)}} d r
$$

where $r_{i}$ is the turning point and $f(r)=r^{2}-\frac{1}{r^{2}}$. Letting $r^{2}=u, \gamma=\sqrt{1+\frac{1}{4} E^{4}}$ and $u=v \gamma-\frac{1}{2} E^{2}$, we obtain

$$
-t_{0}=\int_{r_{i}^{2}}^{\infty} \frac{\frac{1}{2} E u d u}{\left(u^{2}-1\right) \sqrt{\left(u+\frac{1}{2} E^{2}\right)^{2}-\left(1+\frac{1}{4} E^{4}\right)}}=\int_{1}^{\infty} \frac{\frac{1}{2} E\left(v \gamma-\frac{1}{2} E^{2}\right) \gamma d v}{\left(\left(\gamma v-\frac{1}{2} E^{2}\right)^{2}-1\right) \gamma \sqrt{v^{2}-1}}
$$

Now let $v=\cosh x$. The integral becomes

$$
-t_{0}=\frac{1}{2} E \int_{0}^{\infty} \frac{\left(\gamma \cosh x-\frac{1}{2} E^{2}\right) d x}{\gamma^{2} v^{2}-\gamma E^{2} v+\frac{1}{4} E^{4}-1}=\frac{E}{4 \gamma} \int_{0}^{\infty} \frac{\left(2 v-\frac{E^{2}}{\gamma}\right) d x}{v^{2}-\frac{E^{2}}{\gamma} v+\frac{1}{4} E^{4}-1}
$$


Note that the numerator is the derivative of the denominator with respect to $v$. This makes the decomposition into partial fractions easy, and the integral becomes

$$
-t_{0}=\frac{E}{4 \gamma} \int_{0}^{\infty} d x\left(\frac{1}{v-\alpha}+\frac{1}{v-\beta}\right)
$$

where $\alpha=\frac{\frac{1}{2} E^{2}+1}{\gamma}$ and $\beta=\frac{\frac{1}{2} E^{2}-1}{\gamma}$ are the two roots of the denominator of the previous equation. The integrals can now be done by substituting $w=e^{x}$ and writing $\cosh x$ in terms of $w$. The answer is

$$
-t_{0}=\frac{E}{2 \gamma}\left[\frac{1}{\mu_{\alpha}-\nu_{\alpha}} \ln \left(\frac{1-\nu_{\alpha}}{1-\mu_{\alpha}}\right)+\frac{1}{\mu_{\beta}-\nu_{\beta}} \ln \left(\frac{1-\nu_{\beta}}{1-\mu_{\beta}}\right)\right]
$$

where $\mu_{x}=x+\sqrt{x^{2}-1}$ and $\nu_{x}=x-\sqrt{x^{2}-1}$. Using the actual expressions for $\alpha$ and $\beta$ in terms of $E$, we can simplify the answer to the following form:

$$
-t_{0}=\frac{1}{4} \ln \left(\frac{\frac{1}{2} E^{2}-E+1}{\sqrt{1+\frac{1}{4} E^{4}}}\right)-\frac{i}{4} \ln \left(\frac{-\frac{1}{2} E^{2}+i E+1}{\sqrt{1+\frac{1}{4} E^{4}}}\right)
$$

Here we have subtracted the imaginary piece $i \beta / 4$ corresponding to crossing one horizon. One can check that this gives $t_{c}$ for large $E$ and that it goes like $E^{3}$ for small $E$. This shows that there are 3 branches corresponding to the positive $t$ direction starting at 0 on the complex $E$ plane.

Recall that the result (3.11) quoted in Section 3.2 pertained to the time difference between two boundaries, so that for symmetric geodesics, $t=-2 t_{0}$ (defined to be real for real energies $E$ ).

The case of finite mass black hole can also be done exactly, though it is computationally more involved. In particular, following the procedure outlined above for the metric (2.2), we obtain

$$
\begin{array}{r}
-t_{0}(E)=\frac{R^{2}}{2\left(2 r_{+}^{2}+R^{2}\right)}\left\{r_{+} \ln \left(\frac{\left(1+E^{2}\right) R^{2}-2 E R r_{+}+2 r_{+}^{2}}{\sqrt{\left(1+E^{2}\right)^{2} R^{4}+4 R^{2} r_{+}^{2}+4 r_{+}^{4}}}\right)\right. \\
\left.-i \sqrt{r_{+}^{2}+R^{2}} \ln \left(\frac{\left(1-E^{2}\right) R^{2}+2 i E R \sqrt{r_{+}^{2}+R^{2}}+2 r_{+}^{2}}{\sqrt{\left(1+E^{2}\right)^{2} R^{4}+4 R^{2} r_{+}^{2}+4 r_{+}^{4}}}\right)\right\}
\end{array}
$$

Note that for $\bar{t}=\frac{r_{+}}{R^{2}} t$ and $\bar{E}=\frac{R}{r_{+}} E$, in the $\frac{R}{r_{+}} \rightarrow 0$ limit, $-\bar{t}_{0}(\bar{E})$ reduces to (D.6) with barred $t$ and $E$.

Expanding around $E \approx \infty$ yields $t_{c}=-\frac{1}{4 T} \frac{\sqrt{r_{+}^{2}+R^{2}}}{r_{+}}$which can be easily verified by considering radial null geodesic as in (2.7). 
Expanding around $E \approx 0$ yields

$$
-t_{0}(E) \approx \frac{R^{5}}{\left(2 r_{+}^{2}+R^{2}\right)^{2}} E+\frac{R^{5}\left(2 r_{+}^{2}-R^{2}\right)^{2}}{3\left(2 r_{+}^{2}+R^{2}\right)^{4}} E^{3}+\mathcal{O}\left(E^{5}\right) .
$$

In passing we note the intriguing fact that the cubic term flips sign when $r_{+}=\frac{R}{\sqrt{2}}$, which is exactly the same as the transition point when the specific heat becomes negative - that is, at the Hawking-Page transition [62]. Although the physical significance is not clear, it is rather suggestive that these small black holes (which are no longer approximately thermal states in the CFT) have a vastly richer geodesic structure.

The important point, used in Section 3.3, is that $t(E)$ now has the form

$$
t(E) \sim E^{3}-a E
$$

with $a<0$, so that the cubic degeneracy is resolved for Euclidean time.

\section{Appendix E. Analytic structure of $E(t)$}

We have given above a formula for $t(E)$ in the infinite mass black hole limit. This formula defines a Riemann surface over the $t$-plane, and it is amusing to examine its analytic structure. Note that because of formula (2.18) the analytic structure of $E(t)$ is closely related to that of the large $m$ correlation function (modulo extra branch cuts from the $\log$ in (2.18) ). To start, we find the locations of all the branch points of $E(t)$. These must correspond to values of $E$ for which $d t / d E$ vanishes, and a quick examination of $d t / d E$ shows that it vanishes only at $E=0, \infty$.

Now $t$ is periodic with period $i \beta=i \pi$. For $E(t)$, we have first of all the coincident points pole at $\pi i / 2$ (with $\left.E \sim \frac{1}{t-\pi i / 2}\right)$; our previous analysis shows that we have a branch cut at $t=0$ (with $E \sim t^{\frac{1}{3}}$ ), and the $t_{c}$ pole at $\pi / 2$. In fact this pattern of branch cuts and poles repeats itself, with $t^{\frac{1}{3}}$ branch cuts corresponding to points where $E=0$ and thus (looking at the residues of the logs in (3.11) ) occurring at $t=(p+i q) \pi / 2$ with $p+q$ even, and $1 / t$ poles corresponding to points where $E=\infty$ and occurring at $t=(p+i q) \pi / 2$ with $p+q$ odd. Of course, these poles and branch cuts only occur on certain sheets of the Riemann surface. It is not too difficult to understand the sheet structure; the only hard part is seeing what happens at the branch points. There we have 3 sheets. It turns out that two of those (the non- $t_{c}$ branches) do not have any singularities or branch cuts. Moving far in Lorentzian time along these branches corresponds to $E$ spiraling in to one of the fourth roots of -4 , and thus getting far away from $E=0, \infty$, where the poles and branch points occur. Of course, the non- $t_{c}$ branches are the physical ones, so in some sense all the interesting analytic structure is very nonphysical. One possible interpretation for the string 
of $t_{c}$ poles is that they correspond to geodesics bouncing off both the singularities and the boundaries of AdS Schwarzschild multiple times. In order to bounce off a singularity the geodesic has to be spacelike whereas to bounce off the boundary of AdS Schwarzschild it has to be timelike, so this idea is speculative. It is worth noting, however, that the poles on both boundaries predicted above are precisely the locations where the geodesic bounces.

\section{Appendix F. Computing properties of the $t_{c}$ singularity}

In the text we argued that certain information about the black hole singularity is encoded in boundary correlators. In particular there is information in a singularity at $t=t_{c}$ on a secondary sheet of the two point correlator, which at $m=\infty$ has a third order branch point at $t=0$. In this appendix we will show that this singularity can be studied in a computationally effective way, assuming that precise numerical information about the boundary correlator as a function of Euclidean time on the primary sheet is available. Of course such information is not available (for large $\lambda$ ) from direct gauge theory computation at this time. But we feel it is useful to show by example how the process of analytic continuation can be made concrete given well defined gauge theory results.

Specifically we assume that the a number of coefficients in the Taylor expansion of the gauge theory correlator $\langle\phi \phi\rangle(t)$ are known. $L(t)=\frac{-1}{m} \ln \langle\phi \phi\rangle(t)$ has a smooth large $m$ limit so we consider its expansion. For convenience we expand around euclidean antipodal separation, $t=0$ even though $L(t)$ is branched there. The third order character of this branch point is known from our theoretical analysis or would be clear from numerical data. So we write our expansion in terms of $x=t^{\frac{2}{3}}$. For Euclidean time separations the large $m$ evaluation of $L(t)$ from a real geodesic is certainly correct. So we can expand (2.18) and (3.11) to obtain model "numerical" gauge theory data. Stopping at order $x^{20}$ is sufficient to illustrate how things work.

$$
\begin{aligned}
L(x)= & 0.693147-1.36284 x^{2}-0.265333 x^{4}-0.106447 x^{6}-0.0454717 x^{8} \\
& -0.0199415 x^{10}-0.0090257 x^{12}-0.00421708 x^{14}-0.00202004 x^{16} \\
& -0.000984367 x^{18}-0.000485493 x^{20}+\ldots
\end{aligned}
$$

The analytic structure discussed in Section 3.2 and Appendix E implies that the $t_{c}$ singularity of $L$ on the secondary $t$ sheet will appear at $x_{c}=t_{c}^{2 / 3}=1.35128345$. This singularity has the leading behavior $L(x) \sim 2 \log \left(x-x_{c}\right)$.

To find this singularity numerically we use standard techniques. First we differentiate (F.1) so that the leading singular behavior is a simple pole, $L^{\prime}(x) \sim 2 /\left(x-x_{c}\right)$. We then fit (F.1) to a ratio of two polynomials $P$ and $Q$ of degree $M$ and $N, L^{\prime}(x) \simeq P_{M}(x) / Q_{N}(x)$. 
This is called a Padé approximant [63]. The convergence of such approximants is nonuniform, so we select one more or less arbitrarily.

The $[M, N]=[7,12]$ Padé has a pole at $x=1.35128349$ (compared to $x_{c}=$ 1.35128345 ) with a residue of 2.0028 (compared to 2 ) . These results are probably anomalously good. The $[9,10]$ approximant has a pole value of 1.35137 .

So in this limiting case a modest amount of "numerical" data is sufficient to study the singularity. But it is important to ascertain how stable this situation is. In particular, as discussed in Section 4.3, finite $g_{s}$ limits our ability to take $m$ arbitrarily large. We know that at finite $m$ the branch cut at $t=0$ is erased and we can no longer analytically continue to a secondary sheet. As discussed in Section 3.5, a signal of this erasure order one corrections to the order $k$ power series coefficient when $k \sim m$. Here we see that $k=20$ is adequate to determine $L$, so we just need $m>>20$. As also noted in section 3.5 there will be an asymptotic series of $1 / m$ corrections that are branched and can be analytically continued to the $t_{c}$ singularity. These contain interesting physics, as explained in Section 4. However to study the leading behavior they must be handled in some way, perhaps by extrapolation in $1 / \mathrm{m}$. But this may not be done with complete precision so we investigate our ability to still see the $t_{c}$ singularity with some small $1 / m$ correction remaining. In the $x$ variable this correction has a pole at $x=0$ and a higher order pole at $x=x_{c}$. The correct procedure would be to expand around a point $t_{0} \neq 0$ and Padé approximate both $x=0$ and $x=x_{c}$ singularities.

Rather than go through this entire procedure we model the phenomenon by adding the series expansion of a pole at $x=\frac{1}{2}, \frac{1}{m(x-1 / 2)}$ to the derivative of ( F.1 $)$. This pole is much closer to the expansion point $x=0$ than $x_{c}$ is so its power series coefficients grow much faster than those in (F.1 $)$. The ratio of the coefficients at order $n$ is approximately $\frac{1}{m}\left(x_{c} /(1 / 2)\right)^{n} \sim \frac{1}{m}(2.7)^{n}$ At $n=19$ for $m=100$ the $1 / m$ correction is about $10^{7}$ times larger than the main result! Nonetheless the Padé approximant can efficiently separate out these different poles. The [7,12] approximant (with $m=100$ ) yields a pole at $x=1.35169$ with residue 2.0086, as well as the pole at $x=1 / 2$.

As explained in Section 4.1 we expect that the high curvature around the black hole singularity will create a leading $1 / m$ correction of form $1 /\left(m\left(t-t_{c}\right)^{2}\right)$. The series expansion of this correction will eventually dominate over the terms in (F.1). The ratio of terms of order $n$ will be roughly $\frac{1}{m} n^{2}$. There exist techniques to disentangle such confluent singularities in a Padé approximant 63]. But we will content ourselves with showing that this modest rate of growth allows us to ignore $1 / m$ corrections for $m \sim 10^{4}$. The $[7,12]$ approximant with this addition gives a pole at $x=1.35061$ with residue 1.990 . This analysis illustrates how the apparently rather abstract notions of the $m \rightarrow \infty$ limit and subsequent analytic continuation to a singularity on a secondary sheet can be made computationally explicit. Of course the input data, Euclidean gauge theory correlators, do not yet exist! 


\section{References}

[1] V. Balasubramanian, S. F. Hassan, E. Keski-Vakkuri and A. Naqvi, "A space-time orbifold: A toy model for a cosmological arXiv:hep-th/0202187.

[2] L. Cornalba and M. S. Costa, "A New Cosmological Scenario in String Theory," arXiv:hep-th/0203031.

[3] N. Nekrasov, "Milne universe, tachyons, and quantum group" arXiv: hep-th/0203112.

[4] J. Simon, "The geometry of null rotation identifications," JHEP 0206, 001 (2002) arXiv:hep-th/0203201.

[5] H. Liu, G. Moore and N. Seiberg, "Strings in a time-dependent orbifold," JHEP 0206, 045 (2002) arXiv:hep-th/0204168]; "Strings in time-dependent orbifolds," arXiv:hepth/0206182.

[6] S. Elitzur, A. Giveon, D. Kutasov and E. Rabinovici, "From big bang to big crunch and beyond," JHEP 0206, 017 (2002) [arXiv:hep-th/0204189].

[7] L. Cornalba, M. S. Costa and C. Kounnas, "A resolution of the cosmological singularity with orientifolds," arXiv:hep-th/0204261.

[8] B. Craps, D. Kutasov and G. Rajesh, "String propagation in the presence of cosmological singularities," JHEP 0206, 053 (2002) [arXiv:hep-th/0205101].

[9] M. Fabinger and J. McGreevy, "On smooth time-dependent orbifolds and null singularities," arXiv:hep-th/0206196.

[10] A. Lawrence, "On the instability of 3D null singularities," arXiv:hep-th/0205288.

[11] G. T. Horowitz and J. Polchinski, "Instability of spacelike and null orbifold singularities," arXiv:hep-th/0206228.

[12] M. Berkooz, B. Craps, D. Kutasov and G. Rajesh, "Comments on cosmological singularities in string theory," JHEP 0303, 031 (2003) arXiv:hep-th/0212215.

[13] A. Giveon, E. Rabinovici and A. Sever, "Beyond the singularity of the 2-D charged black hole," arXiv:hep-th/0305140.

[14] A. Giveon, E. Rabinovici and A. Sever, "Strings in singular time-dependent backgrounds," arXiv:hep-th/0305137.

[15] C. R. Stephens, G. 't Hooft and B. F. Whiting, "Black hole evaporation without information loss," Class. Quant. Grav. 11, 621 (1994) arXiv:gr-qc/9310006.

[16] L. Susskind, L. Thorlacius and J. Uglum, "The Stretched horizon and black hole complementarity," Phys. Rev. D 48, 3743 (1993) [arXiv:hep-th/9306069.

[17] For reviews see A. Linde, Particle Physics and Inflationary Cosmology Harwood Academic, (1990), and A. H. Guth, "Inflation and eternal inflation," Phys. Rept. 333, 555 (2000) arXiv:astro-ph/0002156

[18] L. Susskind, "The anthropic landscape of string theory," arXiv:hep-th/0302219.

[19] L. Dyson, M. Kleban, and L. Susskind, "Disturbing Implications of a Cosmological Constant," JHEP 0210:011,2002 arXiv:hep-th/0208013. 
[20] N. Goheer, M. Kleban, and L. Susskind, "The Trouble with de Sitter Space," arXiv:hep-th/0212209.

[21] L. Susskind, "The World as a hologram," J. Math. Phys. 36, 6377 (1995) arXiv:hepth/9409089].

[22] T. Banks, W. Fischler, S. H. Shenker and L. Susskind, "M theory as a matrix model: A conjecture," Phys. Rev. D 55, 5112 (1997) arXiv:hep-th/9610043.

[23] J. M. Maldacena, "The large N limit of superconformal field theories and supergravity," Adv. Theor. Math. Phys. 2, 231 (1998) [Int. J. Theor. Phys. 38, 1113 (1999)] arXiv:hep-th/9711200.

[24] S. S. Gubser, I. R. Klebanov and A. M. Polyakov, "Gauge theory correlators from non-critical string theory," Phys. Lett. B 428, 105 (1998) arXiv:hep-th/9802109].

[25] E. Witten, "Anti-de Sitter space and holography," Adv. Theor. Math. Phys. 2, 253 (1998) arXiv:hep-th/9802150.

[26] O. Aharony, S. S. Gubser, J. M. Maldacena, H. Ooguri and Y. Oz, "Large N field theories, string theory and gravity," Phys. Rept. 323, 183 (2000) arXiv:hep-th/9905111.

[27] E. Witten, "Anti-de Sitter space, thermal phase transition, and confinement in gauge theories," Adv. Theor. Math. Phys. 2, 505 (1998) [arXiv:hep-th/9803131.

[28] V. Balasubramanian and S. F. Ross, "Holographic particle detection," Phys. Rev. D 61, 044007 (2000) arXiv:hep-th/9906226.

[29] J. Louko, D. Marolf and S. F. Ross, "On geodesic propagators and black hole holography," Phys. Rev. D 62, 044041 (2000) |arXiv:hep-th/0002111.

[30] J. M. Maldacena, "Eternal black holes in Anti-de-Sitter," arXiv:hep-th/0106112.

[31] P. Kraus, H. Ooguri and S. Shenker, "Inside the horizon with AdS/CFT," arXiv:hepth/0212277.

[32] T. S. Levi and S. F. Ross, "Holography beyond the horizon and cosmic censorship," arXiv:hep-th/0304150.

[33] T. Banks, M. R. Douglas, G. T. Horowitz and E. J. Martinec, "AdS dynamics from conformal field theory," arXiv:hep-th/9808016.

[34] V. Balasubramanian, P. Kraus, A. E. Lawrence and S. P. Trivedi, "Holographic probes of anti-de Sitter space-times," Phys. Rev. D 59, 104021 (1999) arXiv:hep-th/9808017.

[35] D. Kabat and G. Lifschytz, "Gauge theory origins of supergravity causal structure," JHEP 9905, 005 (1999) arXiv:hep-th/9902073.

[36] T. Jacobson, "On the nature of black hole entropy," arXiv:gr-qc/9908031.

[37] L. Susskind and N. Toumbas, "Wilson loops as precursors," Phys. Rev. D 61, 044001 (2000) arXiv:hep-th/9909013.

[38] U. H. Danielsson, E. Keski-Vakkuri and M. Kruczenski, "Black hole formation in AdS and thermalization on the boundary," JHEP 0002, 039 (2000) arXiv:hep-th/9912209.

[39] G. T. Horowitz and V. E. Hubeny, "CFT description of small objects in AdS," JHEP 0010, 027 (2000) arXiv:hep-th/0009051. 
[40] J. P. Gregory and S. F. Ross, "Looking for event horizons using UV/IR relations," Phys. Rev. D 63, 104023 (2001) arXiv:hep-th/0012135.

[41] S. B. Giddings and M. Lippert, "Precursors, black holes, and a locality bound," Phys. Rev. D 65, 024006 (2002) arXiv:hep-th/0103231.

[42] B. Freivogel, S. B. Giddings and M. Lippert, "Toward a theory of precursors," Phys. Rev. D 66, 106002 (2002) arXiv:hep-th/0207083.

[43] V. E. Hubeny, "Precursors see inside black holes," arXiv:hep-th/0208047.

[44] For a review see M. Le Bellac, Thermal Field Theory, Cambridge University Press (1996).

[45] W. Israel, "Thermo Field Dynamics Of Black Holes," Phys. Lett. A 57, 107 (1976).

[46] T. Klosch and T. Strobl, Class. Quant. Grav. 13, 2395 (1996) arXiv:gr-qc/9511081.

[47] J. McGreevy, L. Susskind and N. Toumbas, "Invasion of the giant gravitons from anti-de Sitter space," JHEP 0006, 008 (2000) arXiv:hep-th/0003075.

[48] M. V. Berry and C. J. Howls, "Hyperasymptotics for integrals with saddles," Proc. Roy. Soc. Lond., A434, 657 (1991) .

[49] M. V. Berry, "Uniform asymptotic smoothing of Stokes's discontinuities," Proc. Roy. Soc. Lond., A422, 7 (1989) .

[50] A. Nunez and A. O. Starinets, "AdS/CFT correspondence, quasinormal modes, and thermal correlators in $\mathrm{N}=4 \mathrm{SYM}$," arXiv:hep-th/0302026.

[51] S. H. Shenker, "Another Length Scale in String Theory?," arXiv:hep-th/9509132.

[52] D. Kabat and P. Pouliot, "A Comment on Zero-brane Quantum Mechanics," Phys. Rev. Lett. 77, 1004 (1996) arXiv:hep-th/9603127.

[53] M. R. Douglas, D. Kabat, P. Pouliot and S. H. Shenker, "D-branes and short distances in string theory," Nucl. Phys. B 485, 85 (1997) arXiv:hep-th/9608024.

[54] S. S. Gubser, I. R. Klebanov and A. A. Tseytlin, "Coupling constant dependence in the thermodynamics of $\mathrm{N}=4$ supersymmetric Yang-Mills theory," Nucl. Phys. B 534, 202 (1998) arXiv:hep-th/9805156.

[55] N. Lambert, H. Liu and J. Maldacena, "Closed strings from decaying D-branes," arXiv:hep-th/0303139.

[56] D. Gaiotto, N. Itzhaki and L. Rastelli, "Closed strings as imaginary D-branes," arXiv:hep-th/0304192.

[57] J. M. Maldacena and A. Strominger, "AdS(3) black holes and a stringy exclusion principle," JHEP 9812, 005 (1998) arXiv:hep-th/9804085.

[58] M. T. Grisaru, R. C. Myers and O. Tafjord, "SUSY and Goliath," JHEP 0008, 040 (2000) arXiv:hep-th/0008015.

[59] A. Hashimoto, S. Hirano and N. Itzhaki, "Large branes in AdS and their field theory dual," JHEP 0008, 051 (2000) arXiv:hep-th/0008016.

[60] D. J. Gross and H. Ooguri, "Aspects of large N gauge theory dynamics as seen by string theory," Phys. Rev. D 58, 106002 (1998) arXiv:hep-th/9805129. 
[61] I. Ichinose and Y. Satoh, "Entropies of scalar fields on three-dimensional black holes," Nucl. Phys. B 447, 340 (1995) arXiv:hep-th/9412144.

[62] S. W. Hawking and D. N. Page, "Thermodynamics Of Black Holes In Anti-De Sitter Space," Commun. Math. Phys. 87, 577 (1983).

[63] G.A. Baker and P.R. Graves-Morris, Padé Approximants, second edition, Cambridge Univ. Press, (1995). 